\title{
2005 MRS Spring Meeting Mixes the Aesthetics and Science of Materials Research
}

The 2005 Materials Research Society Spring Meeting presented new and developing areas of materials research as well as established popular topics. Held March 28-April 1 at the Moscone West Convention Center in San Francisco, Calif., the Meeting supplemented the outstanding technical sessions with motifs on art and science, funding and entrepreneurship, a record number of 12 tutorials, and awards. Meeting chairs Joanna Aizenberg (Bell Laboratories/ Lucent Technologies), Oliver Kraft (IMF II, Forschungszentrum Karlsruhe/IZBS, University of Karlsruhe), Neville Moody (Sandia National Laboratories), and Ramamoorthy Ramesh (University of California, Berkeley) classified the 33 technical symposia into six clusters, covering an expanse of materials issues including photonics, nanotechnology, synthesis and fabrication, modeling, biotechnology, and energy. The Meeting included five days of technical sessions and talks, poster sessions, the awards and plenary session, tutorials, special events, an exhibit, and other activities, with about 2800 papers presented in oral and poster sessions to nearly 3100 attendees.

\section{Uncommon Collaborations Joined by \\ Common Interests}

It is rare to find collaborations between scientists and artists, but Etienne Krähenbühl and Rolf Gotthard of Ecole Polytechnique Fédérale de Lausanne have shown how fruitful such a collaboration can be. Eight years ago Krähenbühl, a sculptor, walked into the office of Gotthard, an electron microscopist, and asked if there was a metal that would change color with temperature. The answer was no, but Gotthard did know of something else that might interest a sculptor: a shape-memory alloy. Thus was launched a collaboration that has produced the remarkable works on display at Swissnex, the Swiss cultural center in downtown San Francisco. Krähenbühl and Gotthard held a reception at Swissnex following their talk at the MRS Spring Meeting as part of Symposium X, a lunchtime series of presentations for the technical nonspecialist.

The most striking of the sculptures on display was "Temps Suspendu" ("Suspended Time"), an array of scores of iron cubes suspended from shape-metal-alloy wires. The cubes were cut from compressed train rails, and cast fascinating shadows on the floor. Temperature fluctuations in the room drove contractions in the length of the wires as they returned to their unstretched, "remembered" shapes, and these contractions set the cube array swinging and jangling like so many bells. See more about the collaboration in the sidebar on Symposium X.

Complementing this presentation, the Meeting held-for the first time-a "Science as Art" competition, open to all attendees. Advances in materials characterization approaches are providing unprecedented insight into materials structure and behavior. These approaches underpin all materials discoveries while providing images of a world not visible to the naked eye. While the images created are of great significance to the world of science, the meeting chairs also recognized the aesthetic aspect of the images. Participants of the competition displayed their images throughout the week.

MRS likewise recognizes that another developing opportunity for collaboration resides among materials researchers and

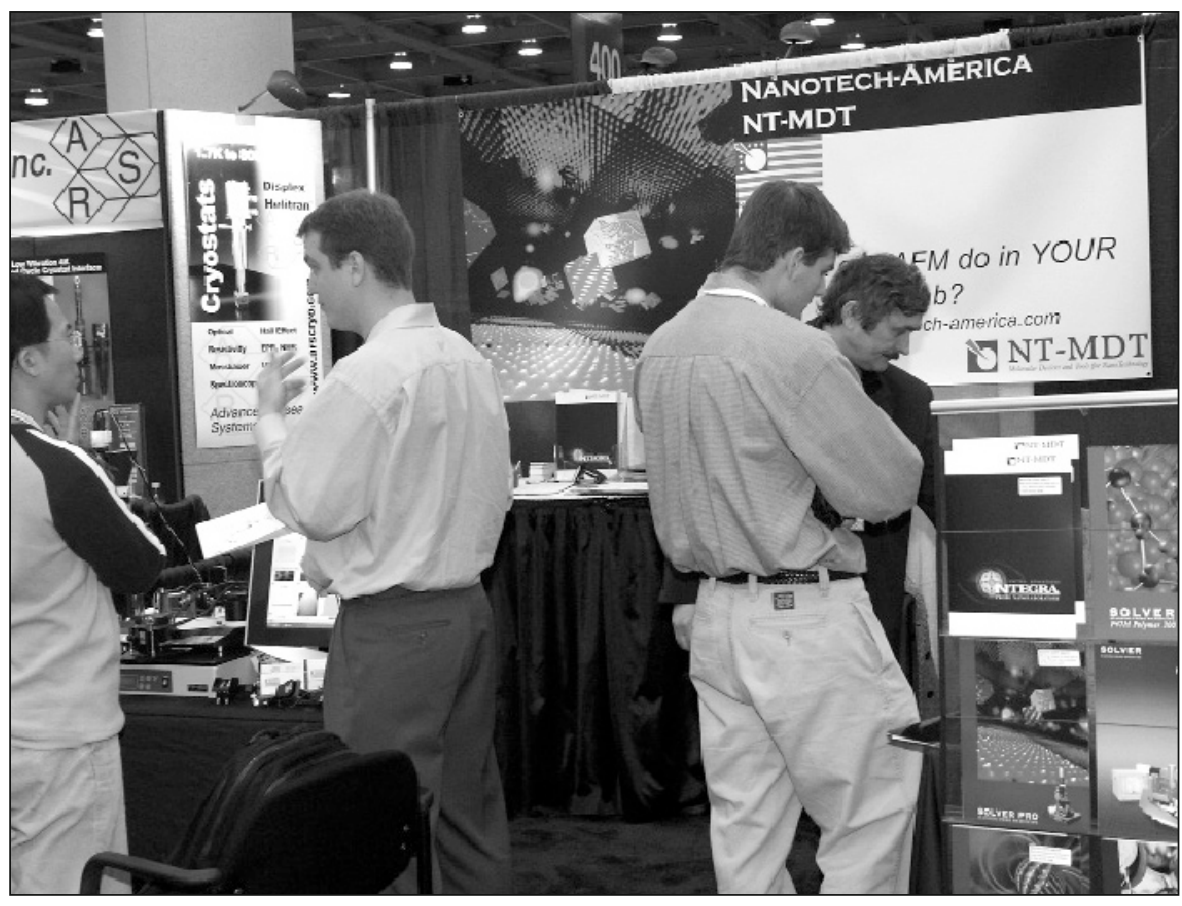

Exhibitors were on-hand to discuss materials research equipment and resources. 
those in the biomedical field. George M. Whitesides, the Mallinckrodt Professor of Chemistry at Harvard University, addressed this issue in his plenary presentation at the Meeting. In recognizing biology and biomedicine as the most important field in the future for humans, Whitesides explored the role of materials science in that area. Current work on developing materials systems for biology and medicine (e.g., stents and artificial hips) is clearly an extension of traditional materials science, he said. Materials research is hierarchically top-down in the attempt to understand macro-properties from the underlying structure. Research in biology is hierarchically bottom-up. Whitesides suggested that rather than build a materials system and then look for a problem to fix, materials scientists should look for problems in biology and then design a materials solution. Thus, materials science should serve, not lead, biology, according to Whitesides.

Whitesides gave specific examples of cases where a biology-related problem was solved using materials, such as cochlear implants and retinal prostheses to aid the senses. In addition, Whitesides said that the materials field could help solve problems in developing countries in a low-cost manner (e.g., very low cost eyeglasses), and that materials solutions could alleviate national security problems.

Whitesides described a changing landscape for materials scientists in which he encouraged materials researchers to put biology in front and build collaborations. These collaborations will lead to funding and venture capital, and will ultimately convert the focus on science into a focus on technology for social benefits, he said. MRS as a society can make a difference, according to Whitesides. The Society can frame and explain the opportunities at the materials/biology interface and also educate its members. MRS could possibly broker collaborations and stimulate seed funding, Whitesides said. He strongly encouraged materials researchers to fur- ther their understanding of the needs in biology research and look for collaborative opportunities in medical schools.

Whitesides's message was corroborated by various speakers in the technical sessions. The human body is a very "harsh, complex, and heterogeneous" environment in which to manipulate magnetic nanoparticles, said Q. Pankhurst (University College, London), while describing challenges to translating nanoparticle materials science into clinically relevant diagnostics and therapies. The use of magnetic nanoparticles is being tested clinically for targeted drug delivery and anti-cancer hyperthermia treatments. As protein purification and MRI contrast agents, first-generation and relatively unsophisticated $\sim 30-300 \mathrm{~nm}$ iron oxide particle aggregates have proven to be commercially successful, said Pankhurst in his presentation. Pankhurst noted that the technology matched the needs of the end user, and he encouraged materials scientists to

\section{ACRONYM KEY}

1D: one-dimensional

2D: two-dimensional

3D: three-dimensional

AFM: atomic force microscopy

AFOSR: Air Force Office of Scientific Research

AFRL: Air Force Research Laboratory

ALD: atomic layer deposition

AMD: Advanced Micro Devices

ANL: Argonne National Laboratory

ANU: Australian National University

ARO: Army Research Office

ASU: Arizona State University

BECON: Bioengineering Consortium

Caltech: California Institute of Technology

CMOS: complementary metal oxide semiconductor

CMP: chemical-mechanical polishing

CNRS/CERI: Centre National de la

Recherche Scientifique/Centre d'Etudes et des Recherches Internationales

CVD: chemical vapor deposition

DFT: density functional theory

DoD: U.S. Department of Defense

DOE: U.S. Department of Energy

EBSD: electron backscattered detector

EELS: electron energy-loss spectroscopy

EPFL: Ecole Polytechnique Fédérale de Lausanne

ETH: Swiss Federal Institute of Technology

FET: field-effect transistor

FIB: focused ion beam

Georgia Tech: Georgia Institute of Technology

GM: General Motors

HP: Hewlett-Packard

HRTEM: high-resolution transmission

electron microscopy
HTS: high-temperature superconductor

IBAD: ion-beam-assisted deposition

IC: integrated circuit

IIT: Indian Institute of Technology

IMRE: Institute of Materials Research and Engineering

INFM: Istituto Nazionale per la Fisica della Materia

IR: infrared

JHU: Johns Hopkins University

LANL: Los Alamos National Laboratory

LCD: liquid-crystal display

LED: light-emitting diode

LIGA: lithography, electrodeposition, and molding

LLNL: Lawrence Livermore National Laboratory

MBE: molecular-beam epitaxy

MEMS: microelectromechanical systems

MIT: Massachusetts Institute of Technology

MOS: metal oxide semiconductor

MOSFET: metal oxide semiconductor

field-effect transistor

MPI: Max Planck Institute

NASA: National Aeronautics and Space

Administration

NCSU: North Carolina State University

NEMS: nanoelectromechanical systems

NIDCR: National Institute of Dental and Craniofacial Research

NIH: National Institutes of Health

NIST: National Institute of Standards and Technology

NRC: National Research Council, Canada

NREL: National Renewable Energy Laboratory

NSF: National Science Foundation

OLED: organic light-emitting diode

ONRL: Oak Ridge National Laboratory
OSU: Ohio State University

PARC: Palo Alto Research Center

PLD: pulsed laser deposition

PSU: Pennsylvania State University

PV: photovoltaic

R\&D: research and development

RFID: radio-frequency identification

RPI: Rensselaer Polytechnic Institute

SEM: scanning electron microscopy

SNL: Sandia National Laboratories

SPM: scanning probe microscopy

SRL-ISTEC: Superconductivity Research

Laboratory-International

Superconductivity Technology Center

STEM: scanning transmission electron

microscopy

STM: scanning tunneling microscopy

SUNY: State University of New York

SWNT: single-walled carbon nanotube

TEM: transmission electron microscopy

TFA: thin film on ASIC

TFT: thin-film transistor

TI: Texas Instruments

UC: University of California

UCLA: University of California, Los Angeles

UCSB: University of California, Santa Barbara

UCSD: University of California, San Diego

UHV: ultrahigh vacuum

UHV-TEM: ultrahigh vacuum transmission

electron microscopy

UIUC: University of Illinois, Urbana-Champaign

UT: University of Texas

UV: ultraviolet

VLSI: very large-scale integration

WPAFB: Wright-Patterson Air Force Base 
interface more with clinicians when advancing nanoparticle technology. Magnetic nanoparticles have also been targeted to specific tissues by externally focusing magnetic fields to spatially localized areas of the body. By using a time-varying magnetic field to impart localized energy to heat and kill cells, hyperthermia treatment represents the most promising recent advance, he said.

Pankhurst also discussed current efforts to exploit natural or "biogenic" sources of nanoparticles in the body. One primary example is that of ferritin, a natural iron storage unit in human tissue. Pankhurst said that such magnetic deposits are abnormally high in the brains of people affected by Alzheimer's disease, a finding that may be used for advanced diagnostic purposes and may indicate a role for anomalous iron homeostasis in the disease pathology. For magnetic nanoparticle technologies to be successful in biomedical applications, experiments must be done in biologically relevant contexts, said Pankhurst. He added that any extrapolation from experiments in solution or idealized systems, as is typically done in materials research, must be performed with much care. Pankhurst gave his presentation in Symposium S; see the summaries on individual symposia later in the report.

DNA is a wonderful material for nanoscale construction, said A. Turberfield (Oxford) in his Symposium K talk about nanomachines made from DNA. Turberfield gave his presentation within a cluster of technical sessions devoted to biological and molecular materials, continuing MRS's emphasis on this burgeoning area. DNA is a structural material whose assembly can be programmed by making use of its information carrying capability. Its hybridization can also be used as an energy source for molecular devices. Turberfield described recent work on 3D nanofabrication, DNA-templated protein crystals, and molecular machinery. Specifically, he described the formation of DNA tetrahedra that can be formed rapidly using self-assembly in less than a minute. He covered recent progress toward the construction of a free-running synthetic molecular motor using DNA and indicated that researchers were very close to achieving it.

Although nanoscale fabrication techniques seem to be well-developed for materials such as silicon, similar techniques are not nearly as well developed for interfacing materials with biology. Such techniques would be advantageous for the fabrication of nanoscale polymer coatings that repel proteins. So far, only metal coatings (i.e., gold) satisfy this tax-

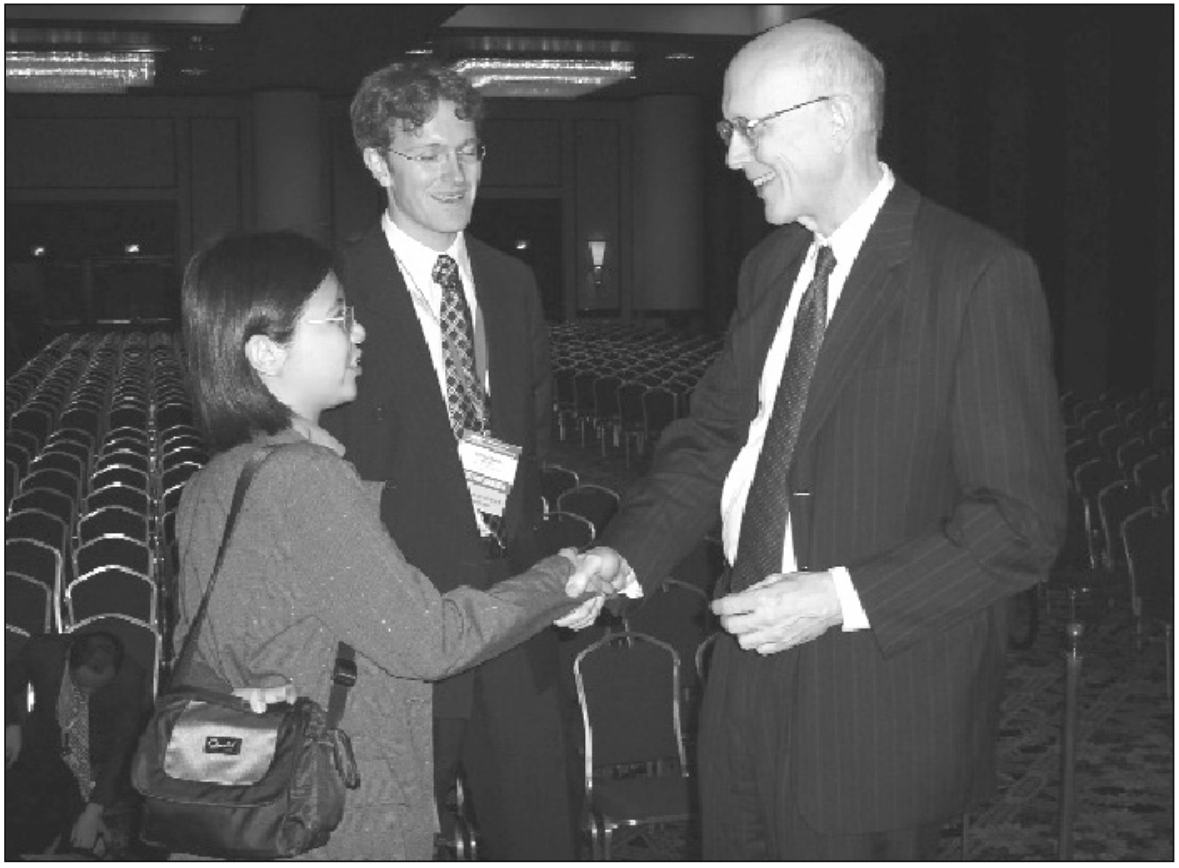

George M. Whitesides of Harvard University greets other researchers following his plenary address, in which he encouraged materials/biomedical research collaborations.

ing requirement, but these coatings operate only for about one week. A. Chilkoti (Duke Univ.), in Symposium M, proposed an alternative strategy based on surface-initiated polymerization. Such a technique leads to dense polymer brushes, while no bulk polymers are generated. The approach allows for the creation of very fine surface structures. Chilkoti also presented the possibility of using enzymes to develop new nanolithography techniques. He demonstrated a method to excavate DNA at the mesoscale using an adsorbed enzyme. In this approach, an enzyme, DNAse I, locally deposited on a homogeneous layer of an oligonucleotide substrate, was activated to locally digest immobilized DNA with nanoscale precision, resulting in nanotrenches of DNA that are biochemically carved by the enzyme into the surface. He demonstrated methods that can be used to "write" nanostructures (e.g., channels) of DNA on the surface and to create scaffolds in the $\mathrm{z}$ direction which other components can be snapped onto by molecular recognition.

In another Symposium M talk on the materials/biology interface at the nanoscale, J.D. Ballard (RPI) and colleagues have found that the adhesion properties of various cells may be a function of the size of the characteristic dimensions of the surface nanostructure. Ballard reported that the use of $100 \mathrm{~nm}$ Si nanoparticles on a substrate significantly reduced the adhesion of osteoblasts to the substrate while increasing adhesion of fibroblasts to the substrate; however, smaller nanoparticles led to better adhesion of the osteoblasts. This behavior is based on integrin protein complexes that connect the extracellular environments with intracellular cytoskeleton. The control of cell adhesion may find applications in medicine and biotechnologies.

In Symposium L, M.D. Morris (Univ. of Michigan) described the molecular response of bone matrix collagen and bone minerals to mechanical loading, which is important in forming a complete picture of fracture in bone materials. However, few experimental techniques allow insight into atomic-scale mechanisms of deformation. Raman spectroscopy was used to study the role of microcracks in such deformation of bone-like materials that is induced by mechanical means such as indenters. The results show that damage occurs to the matrix material at the edges of the indenter, but no changes occur at the center of an indent. Morris concluded that at the edges, shearing forces lead to the rupture of cross-links in the bone matrix. SEM imaging reveals shear bands after deformation, which agrees with known results that the matrix material is much stronger in compression than in shear. Morris showed that cross-linking is essential for maintaining bone strength and stiffness. 


\section{Materials Innovations Advance Key Areas of Research}

The ability to microfabricate optical materials has major consequences for the future of photonic circuitry, explained S. John (Univ. of Toronto) in Symposium A. While discussing the nature of photonic bandgaps and their analogy to solidstate electron bandgaps, John presented examples such as a silicon inverted-opal material (an array of holes in a siliconbased matrix) that displays a 3D bandgap at $1.5 \mu \mathrm{m}$. Using a direct laser-writing technique, John constructed essentially arbitrary Si-based microstructures with tunable bandgaps. The physics of photonic bandgaps may find its most useful application in photonic circuits, that is, devices that use bandgaps to confine and guide light. While it is possible to construct photonic circuits using 2D photonicbandgap materials sandwiched between confining planes, these devices are highly sensitive to small imperfections and can be very lossy. As a result, robust photonic circuits will need to be based on 3D photonicbandgap materials. As examples, John presented systems with frequency-selective extraction for frequency-multiplexed optical signals and waveguides in photonicbandgap heterostructures that display transmission speeds of up to $25 \%$ of vacuum light speed.

One of the elements likely to be required for photonic circuits is a switchable, spectrally dependent reflection device. T. Bunning (AFRL) described his group's work with periodic liquid-crystal nanostructures that can controllably transmit or reflect a narrow wavelength band. Using holographic photopolymerization, Bunning fabricated devices with alternating rich and poor layers of droplets of azobenzene chromophore nanoparticles. The nanoparticles tend to self-orient within the droplets, with the result that polarized incident light experiences a significantly different index of refraction in the droplet-rich layers as compared with the underlying polymer. The alternating layers form a photonicbandgap material that reflects a narrow bandwidth of incident light. When the device is illuminated with a UV pulse, the orientation is relaxed, reducing the index contrast between the nanoparticle-rich layers and the polymer and eliminating the bandgap effect. The device can be switched back to the bandgap state by using an optical pulse. The state persistence is on the order of hours, and the switching times are tens to hundreds of microseconds, Bunning said. He is currently exploring this technique on devices whose natural state is transparent.

Semiconductor quantum dots have enormous potential in a variety of application areas, including as single-photon sources for quantum cryptography and computation and for in vivo biological imaging. However, many aspects of quantum dot emission remain poorly understood, particularly the peculiar "blinking" emission effect in which dots intermittently turn on and off. Studies of this blinking phenomenon are hampered by the difficulty of measuring the exact timing of emission changes from single dots. In Symposium J, K. Zhang (UC-Berkeley) presented an algorithm to quantitatively reconstruct the timing of dot emission, photon by photon. Using a frequency-doubled modelocked laser, Zhang and collaborators excited single quantum dots at $490 \mathrm{~nm}$ and counted emitted photons. For dynamics as short as $1.8 \mathrm{~ms}$, the algorithm accurately extracted the timing of single-photon emissions. The timing data did not support a discrete-emission-state model for the blinking effect, and lifetime measurements showed a nonlinear correlation between emission intensity and luminescence lifetime. Zhang and collaborators have proposed a model of continuously distributed bright states in single quantum dots to explain their data.

Heat can be transformed into electricity in thermionic energy conversion. Using a hot electron emitter and a cooler collector separated by a vacuum gap, a voltage can be produced. One advantage of such systems is that there are no moving parts, so for naval or space missions, for example, this would be a low-maintenance energy choice. In Symposium T, F.A. Koeck (NCSU) described developments in this area. Conventionally, this process relies on flat, metal-based electron emitters that required high operating temperatures, and space-charge effects limit current and efficiencies. Koeck described two diamondbased systems that offer reduced operatingtemperature regimes and more efficient operation. Deposited by microwave-assisted CVD, diamond films doped with nitrogen showed that uniform emission is possible with a lower emission barrier due to negative electron affinity. A voltage was detected at the relatively low temperature of $580^{\circ} \mathrm{C}$, with strongly increasing emission with temperature. Also, sulfur-doped nanocrystalline diamond films can be used. Field-enhancement-based emitters can reduce space-charge effects by providing a means of efficient carrier release into the vacuum gap.

While the move toward nanoelectronics continues, so does progress in the opposite direction, toward large-area electronics. "Let's ride this trend," said S. Wagner (Princeton) in his presentation on flexible, conformal, and elastic electronic surfaces in Symposium H. The display industry is developing tools for fabricating macroelectronics, with the expectation of reducing costs of large-area electronic surfaces in the store to $\$ 1000 / \mathrm{m}^{2}$ in $5-10$ years, he said. While that industry is focused on flat displays, flexible electronics can benefit by building on those successes, and shaping them afterward-literally. Bending, conformal shaping, and stretching have already been demonstrated in the laboratory, opening possibilities toward surround displays, automobile dashboards made of integrated circuits, and prosthetic skin. Focusing on hydrogenated amorphous silicon thin-film transistors, Wagner addressed some of the issues needed to feed these developments. He focused on what happens in the elastic regime as a large, flat surface is bent, how fracture can be prevented, and how the "safe" regime can be extended. One way to reduce fracture is to place stiff thin-film devices on islands attached to a deformable substrate. The islands stay intact while the strain is transferred to the material below, which deforms. To prevent fracture, interconnects can be made out of plastic or elastic materials. To prevent crack propagation, a soft passivating coating can be added. To further extend the safe regime, a system of allmoldable materials can be used, thus eliminating the need even for islands. There are many possibilities, and "getting there makes us walk through an experimenter's paradise," Wagner said.

In another presentation in Symposium H, M. Hack (Universal Display Corp.) gave a glimpse of what the future of display technology might hold. Conventional fluorescent OLEDs (PHOLEDs) tend to be limited because only excitons in the singlet spin state can emit light. In his PHOLEDs, Hack uses heavy-metal ion doping to permit the triplet-state excitons to emit in addition to the singlet spin states, increasing efficiency and reducing power requirements, which in turn lowers the operating temperatures and extends the lifetimes of the devices. Red PHOLEDs at his company have lifetimes of $25,000 \mathrm{~h}$ or more. Among the challenges in developing robust, flexible OLED screens, Hack said, is the need for extreme substrate smoothness (better than $10 \AA$ ) and a protective layer that shields the sensitive OLEDs from oxygen and moisture. Hack predicts that within a few years, companies will place thin-film OLED display screens on the U.S. market.

"Failure of doping semiconductors is basically their kiss of death," began A. Zunger in Symposium E with his pre- 
sentation on doping strategies and doping rules in difficult-to-dope semiconductors. A number of well-known semiconductor systems are difficult to dope (e.g, n-type doping of diamond and Ga-chalcopyrites as well as $p$-type doping of $\mathrm{ZnO}$ and any doping of $\mathrm{MgO}$ ). Such failures have been treated in the literature as isolated cases. Yet, Zunger illuminated common features and common rules concerning these difficultto-dope semiconductors. The most serious issue is the spontaneous formation of compensating, "killer defects" that cause the carrier "to commit suicide," as Zunger put it, and which cannot be easily circumvented. Each semiconductor has an intrinsic "pinning level" that measures its propensity to create those killer defects. In a given class of semiconductors such as III-V or II-VI, for example, $n$-doping is easy, when the conduction-band minimum is below the pinning level, and difficult when above, Zunger said. The corresponding situation applies to $p$-doping: when the valence-band maximum is above the pinning level, $p$-doping is no problem, but very difficult when lower. Zunger offered, then, a number of practical doping rules designed to kill the killer defects. One option could be the use of cluster doping of, for example, $\mathrm{ZnS}$ or ZnSe with GaAs, showing that certain cluster stoichiometries are stable and are promising candidates for effective doping of otherwise difficult-to-dope semiconductors. His rules provide Paulingesque approaches to design doping in difficult-to-dope cases.

"What are the relevant length scales for thin-film plasticity?" asked J.J. Vlassak (Harvard) in Symposium BB. Metal thin films, as used in ICs, hard coatings for wear protection, and MEMS are typically on the order of $1 \mu \mathrm{m}$ thick. In this range, the film microstructure and surrounding barrier layers can impose interesting constraints on strengthening mechanisms in the film, thereby causing large deviations in film behavior from that of the corresponding bulk material. Canonical models, such as those described by Bauschinger over a 100 years ago, seem to describe only one dimension of the phenomenology in these complex metal thin films with many dislocations and grain boundaries.

In an effort toward answering his question, Vlassak described both simulation and experimental results for stress-strain curves of electroplated and sputtered copper thin films. He introduced a technique of testing the mechanical properties of these films, called the plane strain bulge test, that can be performed isothermally, in contrast to traditional substrate curvature techniques. In combination with micro- structural studies, bulge test results reveal that twin boundaries can act effectively as grain boundaries. Furthermore, discrete dislocation dynamic simulation results in the films approached experimental results, but a significant gap between theory and experiment still exists. The close coupling between experiment and model as illus-

\section{Graduate Students Receive Gold and Silver Awards}

During the awards ceremony held on March 30 at the 2005 Materials Research Society Spring Meeting, graduate student finalists received Gold and Silver Awards.

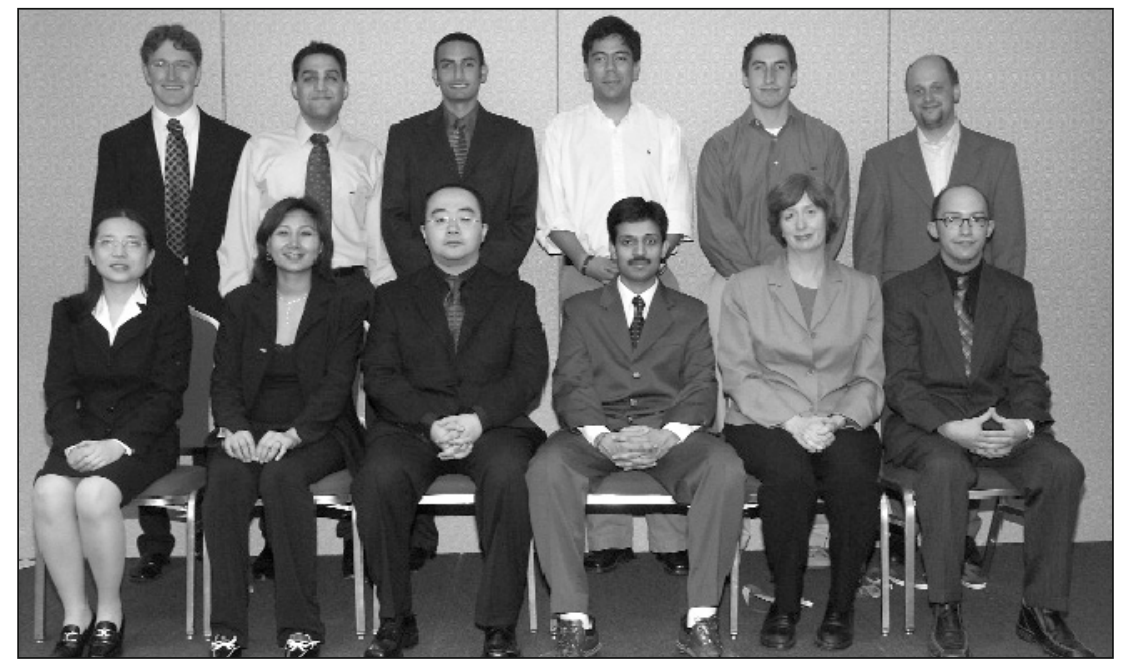

Gold Graduate Student Awards went to (front row, left to right) Yongqing Huang (University of Illinois, Urbana-Champaign), Chanda R. Yonzon (Northwestern University), Yue Wu (Harvard University), Raghuveer S. Makala (Rensselaer Polytechnic Institute), Helen K. Baca (University of New Mexico), and Robert P. Sebra (University of Colorado, Boulder); and (back row, left to right) Benjamin J. Wiley (University of Washington), Ammar M. Nayfeh (Stanford University), Joseph F. AuBuchon (University of California, San Diego), Juan C. Idrobo (University of California, Davis),

Daniel S. Gianola (Johns Hopkins University), and Daniel Abou-Ras (ETH Zurich).

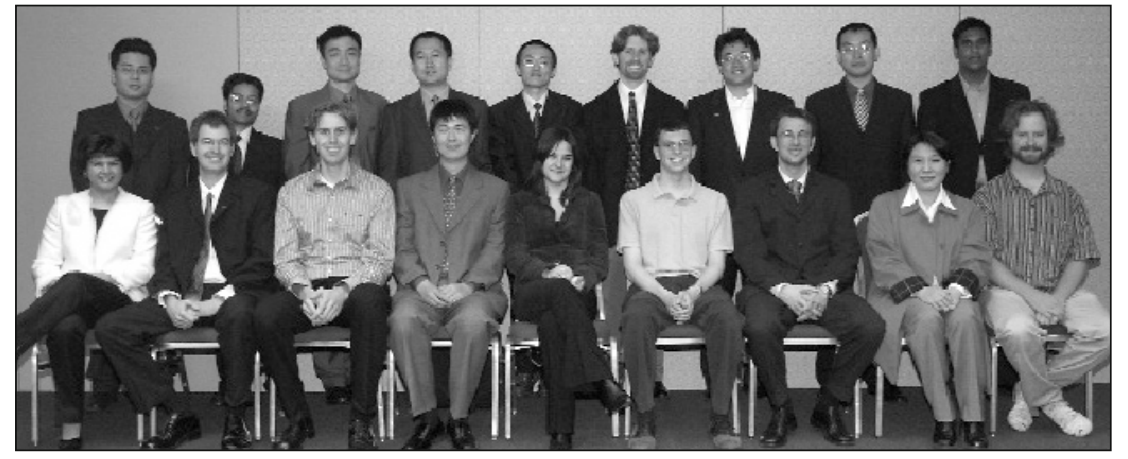

Silver Graduate Student Awards went to (front row, left to right) Shabnam Virji (University of California, Los Angeles), Paul Zaslansky (Weizmann Institute of Science), Igor M.P. Aarts (Eindhoven University of Technology), Xiaowei Teng (University of Rochester), Cinzia Casiraghi (Cambridge University), Michael J. Hetzer (Ohio State University), Patric Gruber (Universität Stuttgart), Sung Yeun Choi (University of Toronto), and Erik J. Menke (University of California, Irvine); and (back row, left to right) Sehoon Yoo (Ohio State University), Mayur S. Valipa (University of Massachusetts, Amherst), Qiming Li (University of New Mexico), Jie Zheng (Georgia Institute of Technology), Feng Tao (Princeton University), Robin S. Friedman (Harvard University), Bentao Cui (University of Minnesota), Zhiwei (Steven) Shan (University of Pittsburgh), and Krishanu Saha (University of California, Berkeley). Not shown are Regis J. Kline (Stanford University) and Oren Tal (Tel Aviv University). 
trated in Vlassak's work may bridge this gap and answer his question, thereby allowing researchers to build detailed mechanistic models with the appropriate spatial resolution.
The recent successful Mars rover landings were a victory for spaceflight technology and for planetary science. However, it is clear that future landings of robotic and staffed missions will require much better landing precision than what is currently possible. Velocity and position measurement uncertainties during descent, as well as the influence of wind, mean that landing precision is no

\section{A Day of Tutorials Prepares Attendees for Technical Talks}

The meeting chairs for the 2005 MRS Spring Meeting offered a record number of 12 tutorials, which were held before the technical sessions opened. A range of topics were presented, including silicon in large-area, flexible electronics; waferlevel packaging; semiconductor heterojunctions; bioMEMS; carbon nanotubebased nanotechnology; and hydrogen storage materials.

In a special tutorial, a group of young researchers-Daniel Abou-Ras (ETH Zurich), Jennifer Heath (Linfield College), Xiangxin Liu (Univ. of Toledo), and Thorsten Rissom (Hahn-Meitner Institute, Berlin)—designed their presentation for young scientists in order to generate open discussion and questions among young researchers. The topic addressed in this inaugural endeavor was characterization techniques of thinfilm solar cells, as part of Symposium F on thin-film compound semiconductor photovoltaics. Bill Shafarman (Univ. of Delaware) gave a brief introduction. He went over the achievements and limitations of crystal Si for solar cells and delineated the performance and manufacturing capabilities of different kinds of thin-film solar cells-a-Si and alloys, CdTe, and CuInSe ${ }_{2}$ and alloys. His colleagues were then available to explain how specific characterization techniques were being used to address the issues raised through thin-film PVs. The tutorial generated a lot of discussion during the session, fulfilling one of its major goals.

From basic scientific studies to drug discovery, neural probes, and tissue engineering, bioMEMS is finding its place in materials research. The tutorial on bioMEMS, held at the start of Symposium J, focused on micro- and nanofabrication, as well as the biocompatibility of tiny devices that interface with living tissue. With fabrication technology matching biological length scales, bioMEMS has moved to center stage. Kevin Turner (MIT) began the tutorial with an overview of the fabrication technologies typically used in the construction of bioMEMS. He started with traditional technologies such as surface and bulk micromachining of silicon and quickly progressed to methods for fabricating polymer microsystems

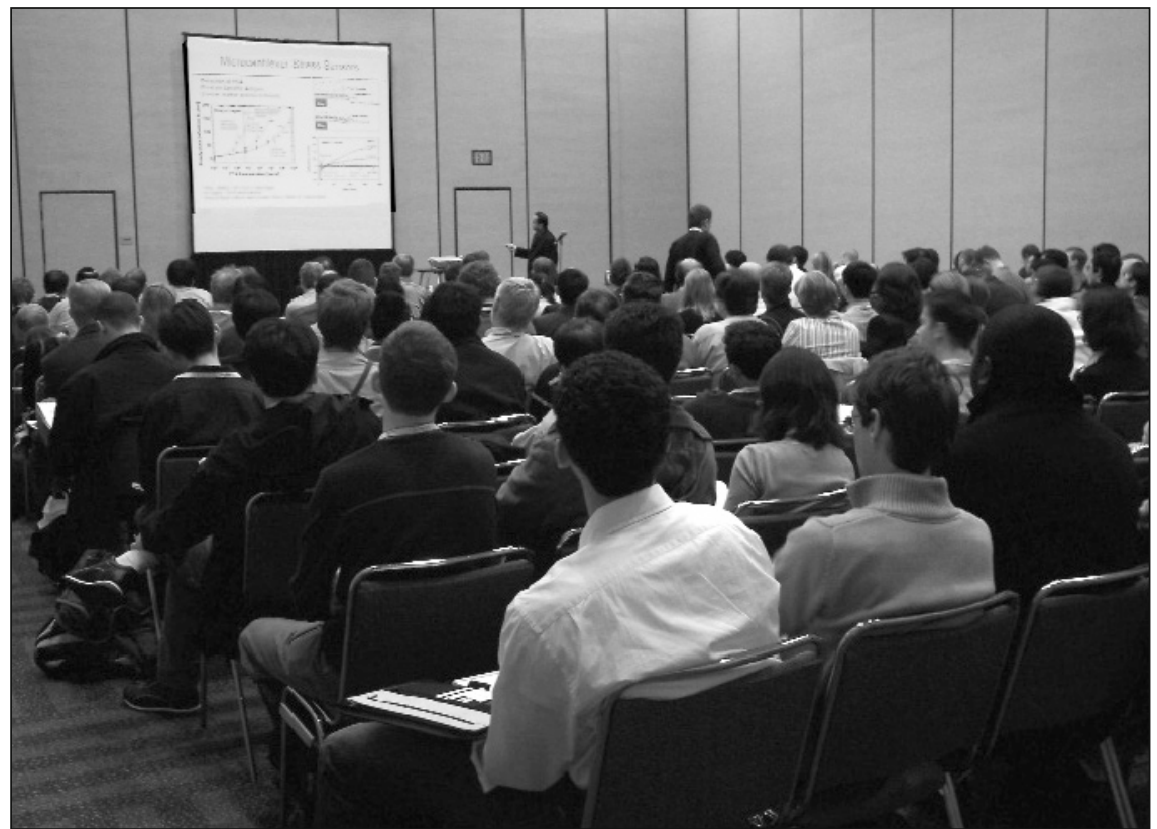

A record number of 12 tutorials were offered at the 2005 MRS Spring Meeting.

and patterning cellular materials, covering a range of additive and subtractive methods. While silicon micro- and nanofabrication is very advanced, other alternatives offer optical transparency (glass) or cost savings (polymers). Rashid Bashir (Purdue) covered the next part of the tutorial, highlighting devices. Numerous biosensors and microfluidic systems have been developed for a range of applications from protein and DNA detection to drug delivery. The final section of the tutorial focused on selection of materials for bioMEMS and biocompatibility, covered by David A. LaVan (Yale).

If there is going to be a "hydrogen economy," there needs to be hydrogen, and a way to store it. Such was the focus of the tutorial complementing Symposium GG, Materials for Hydrogen Storage and Production. In covering critical aspects of hydrogen storage, GholamAbbas Nazri (GM R\&D Ctr.) particularly focused on the chemical absorption of hydrogen for reversible systems, but he also addressed physical adsorption using porous materials and composite mate- rials combining physical and chemical processes. The challenge in this field is in achieving high storage capacity, low weight, and rapid reversible capture and release of hydrogen, so that energy efficiency can be realized and consumers can tolerate refueling. Currently, most hydrogen is produced from fossil fuels and other organic matter, so another challenge is in efficiently extracting the hydrogen rather than turning the starting materials directly into electricity. However, other techniques for obtaining hydrogen are being studied, such as electrolysis. The tutorial concluded with Ping Chen's (Natl. Univ. of Singapore) presentation of recent discoveries and new approaches in light metal-N-H complexes, binary nitrides and imides, ternary complexes, and other systems. Some of these materials showed promising storage capacities and kinetics, with plenty of room for optimization through catalysts, additives, and other parameters.

For more on the tutorials, view news and highlights from the 2005 MRS Spring Meeting on the MRS Web site at www.mrs.org. 
better than $100 \mathrm{~km}$ on planetary bodies with atmospheres, and $1 \mathrm{~km}$ on those without. Since much of the interesting science on Mars and other bodies is in rugged areas where even $1 \mathrm{~km}$ is far too large an error, new landing guidance concepts are needed. In Symposium FF, R. Blaize of NASA Langley described efforts to create an end-to-end autonomous landing system incorporating lidar (i.e., laser radar) to provide the data necessary for safe pinpoint landing on Mars, given the high-precision range and velocity of the lander and the atmospheric winds. Simulations of this system demonstrate that it should be possible to measure the position of a lander during its descent to within $5 \mathrm{~cm}$, and its velocity to within $2 \mathrm{~cm} / \mathrm{s}$. These measurement capabilities should result in landing errors well within the next-generation requirements of only a few kilometers in atmospheric bodies, and sub-kilometer accuracy on those without atmospheres. Not only will this help to place landers accurately near high-scientific-value targets, but it will also protect them from striking large objects. Blaize said that improved landing accuracy would clearly be necessary for a future era of staffed bases on the moon and Mars, where a space-drop delivery of supplies would have to arrive near a base, but not on it.

For further details on the technical content of the Meeting, see the following symposium summaries. Proceedings are available on-line at www.mrs.org.

\section{Photon Takes Center Stage in Connection with Amorphous and Nanocrystalline Silicon, Saluting} Einstein, and the World Year of Physics (See MRS Proceedings Volume 862)

This year, Symposium A on Amorphous and Nanocrystalline Silicon Science and Technology-2005, in its 22nd year at the MRS Spring Meeting, organized three sessions in celebration of the 2005 World Year of Physics and Einstein's modern definition of the photon, as well as other topical sessions.

Among the highlights of the symposium was the opening session on pioneers in the use of photons to study films. W. Jackson (HP) surveyed the beginnings of photothermal deflection spectroscopy, G. Cody (Rutgers/Exxon) gave a historical overview of optical absorption and the Urbach edge in amorphous semiconductors, and L. Ley (Univ. of Erlangen) summarized the seminal contributions of photoelectron spectroscopy to the field. These early advances shaped the subsequent research directions pursued in this field. In a session introduced this year on photonic devices, S. John (Univ. of Toronto) described photonic-bandgap materials accompanied by spectacular video animations, and G. Moddel (Phiar Corp./ Univ. of Colorado) described emerging metal-insulator-metal devices for terahertz detection. In situ optical characterization and growth was described by H. Fujiwara (National Inst. of Advanced Science and Technology, Tsukuba), D. Levi (NREL), and W. Kessels (Eindhoven Univ. of Technology) in a special session.

The symposium was marked with lively contributions in solar-cell-related areas, including the role of hydrogen, metastability, in situ characterization, atomistic materials simulations, and growth. Over the past few years, there has been an increasing number of contributions on microcrystalline silicon, nanostructures, epitaxy, and grain growth. Applications ranging from the mature thin-film transistors, solar cells, and image sensors to the emerging nanocrystal-based devices were highlighted in various device sessions. As in previous meetings, the metastability session brought unexpected results and spirited discussion. A tutorial preceding the symposium by J.P. Conde (Inst. Superior Técnico, Lisbon) and S. Wagner (Princeton) provided a summary of the fundamentals and the state of the art in applications.

Symposium Support: NREL, PARC, United Solar Ovonic, and Voltaix.

\section{Reliable and Novel Interconnects Sought to Support Continued Circuit Scaling \\ (See MRS Proceedings Volume 863)}

The continued scaling of physical dimensions in the IC industry necessitates the introduction of novel materials, processes, integrations, and packages. Symposium B on Materials, Technology, and Reliability of Advanced Interconnects brought together leading modelers and experimentalists to address the plethora of process and reliability issues associated with depositing, characterizing, and integrating novel and existing barriers, metals, and ultralow- $\kappa$ dielectrics into reliable, high-performance interconnects that can be robustly packaged.

The importance of interface integrity and adhesion in low- $\kappa$ dielectric integration was presented by R. Dauschkardt (Stanford), while the issue of processinduced dielectric damage was elucidated by T. Ryan (AMD). Their talks were followed by several contributions highlighting pore-sealing methods for low- $\kappa$ films. Channel cracking is a significant reliability concern for the integration of organosilicate low- $\kappa$ dielectrics, as was discussed in detail by T. Shaw (IBM) and T. Tsui (TI). Shaw showed that the driving force for crack propagation in patterned structures is significantly larger than in field regions, while Tsui focused on the nefarious effects of compliant underlayers.

Mechanical stress was an underlying theme in this symposium, for both dielectrics and metals, and the symposium included a joint session with Symposium O. From the metals perspective, P. Ho (Univ. of Texas) and Y.-C. Joo (Seoul Natl. Univ.) provided a fundamental understanding of the issues of stress and stress relaxation in $\mathrm{Cu}$ films and lines encapsulated in low- $\kappa$ dielectrics. The symposium also included inspiring presentations on electromigration, leakage, and time-dependent dielectric breakdown, as well as thermal and mechanical fatigue. Also, a review of current packaging issues such as packaging and solder reliability and of the adhesion of polymer underfill was covered.

Symposium Support: AMD, Applied Materials, TI, and Tokyo Electron America.

\section{Update on HTS Examines \\ Applications, Processing, and Characterization}

(See MRS Proceedings Volume 868E)

Symposium $C$ provided a forum to update the HTS community on the manifold advances in the multidisciplinary fields that make practical superconductivity applications possible. Topical areas included metal oxide heteroepitaxy, texture development, ion-beam processing, electromagnetic measurements, nanoengineering, and atomic-scale characterization of electronic oxides. Successful application was evident in the summaries on long-length superconducting $\mathrm{YBa}_{2} \mathrm{Cu}_{3} \mathrm{O}_{y}(\mathrm{YBCO})$ and $\mathrm{MgB}_{2}$ wire by Y. Yamada and T. Izumi of SRL-ISTEC in Japan, V. Selvamanickam (SuperPower), T. Kodenkandath (American Superconductor), and G. Grasso (INFM, Italy).

A number of talks highlighted advancements in the past year. P.N. Arendt (LANL) detailed the texturing mechanism that occurs during IBAD of $\mathrm{MgO}$ films. Another part of the symposium dealt with atomistic processes in the heteroepitaxial growth of metal oxide films. Md.S. Bhuiyan (ORNL) and P. Clem (SNL) described buffer layers with "tunable" lattice parameters. Interface engineering in HTS composites was shown by D.H.A. Blank (Twente) to be an effective way to control superconducting properties. Electron microscopy methods for probing the atomic-scale properties of interfaces in metal oxide films were described by N.D. Browning (UC-Davis). Many deposition 
processes to produce epitaxial metal oxide buffer layers or high-critical-current-density YBCO layers in HTS composites were discussed. S.R. Foltyn (LANL) presented results for HTS multilayers made by PLD that are capable of carrying up to $1400 \mathrm{~A}$ (4 MA $\left./ \mathrm{cm}^{2}, 75 \mathrm{~K}, \mathrm{SF}\right)$ in a HTS multilayer structure $3.5 \mu \mathrm{m}$ thick and $1 \mathrm{~cm}$ wide. Advances in film deposition now allow researchers to nanoengineer defect structures into the superconducting films, which improves performance in applied magnetic fields. A sampling of these approaches are rare-earth substitutions presented by K. Matsumoto (Kyoto); rareearth ion size and variance effects and nanoparticle additions by J. MacManusDriscoll (Cambridge) and P.N. Barnes (WPAFB); rare-earth, ultrafine multilayers by T.G. Holesinger (LANL), and nanolines (bamboo structure) by Yamada (SRL-ISTEC).

Symposium Support: ANL, LANL, ORNL, and Univ. of Wisconsin-Applied Superconductivity Ctr.

\section{Monolithic Instruments Integrate Electronics and Function into Solid-State Device \\ (See MRS Proceedings Volume 869)}

The first session of Symposium D on Materials, Integration, and Technology for Monolithic Instruments dealt with performance imagers in TFA technology using elevated thin-film photodiodes of amorphous silicon as detectors on top of conventional CMOS circuits, thus allowing the fabrication of complex monolithically integrated instruments for numerous detection applications. N. Wyrsch (Inst. of Microtechnologies, Switzerland) gave an assessment of the present status of the technology, while other speakers focused on specific device issues, in particular, ways to reduce the dark current of the detectors and to improve transient behavior. J. Sterzel of Jena-Optronik, a Germany-based technology company focusing on imagers for space vehicles, talked about detection limits of such imagers. Sterzel provided insight into the complex task of describing crucial performance parameters, taking into account all system components and the specifics of the device architecture resulting from the integration of the detectors on top of a CMOS chip. Sensitivity as well as temporal and local noise was among the important issues discussed to improve the performance parameters of high-end imagers. P. Catrysse (Stanford) outlined innovative schemes on the integration of subwavelength metal optics into CMOS technology, opening up new ways to replace conventional Bayer pattern color filters by integrating metal grating structures acting as color filters. Several speakers focused on specific issues regarding device design for the integration of detectors and waveguides for different wavelength ranges on an integrated circuit and on process compatibility issues. Among them were multispectral diodes that enable a biascontrolled shift of the quantum efficiency as well as novel schemes to fabricate IR photodetectors and waveguides monolithically integrated with Si-based photonic circuits.

$R$. Thewes (Infineon) presented groundbreaking work on DNA detection systems incorporating a monolithic CMOS device with chemical wells that detect charge produced by redox reactions by using a specially design transimpedance amplifier. D. Martin (Univ. of Michigan) discussed the processing challenges in creating liquid chemical sensing systems. N. Lewis (Caltech) showed the development of very simple yet sensitive gas chemical detection systems through the use of resistive polymer array devices called electronic noses. These devices mimic mammalian olfactory senses. He showed that it is possible to reliably detect the difference between volatile molecules such as alkane isomers over many days using such sensors, and that for some compounds, these devices can have parts-per-billion detection limits. K. Suslick (UIUC) presented work on metalloporphyrin materials he has developed to perform olfactory-like sensing using colorimetric changes in the films. His materials can be developed into arrays to detect differences between complicated multicomponent samples such as those found in food.

M. Handschy (Displaytech Inc., Colorado) presented an historical overview of LCDs on silicon. Originally, the LCDs were being considered for optical computing, but they were eventually incorporated into viewfinders for cameras. He said that although liquid-crystal technology is slower than conventional display technologies, it consumes less power at high frame rates. E. Foca (ChristianAlbrechts-Univ. of Kiel, Germany) presented studies in lenses fabricated using photonic-crystal technology and refractive indexes of less than one. X. Yu (Stanford) presented results from growing $\mathrm{GaAs}$ on silicon using Ge as a buffer layer. The luminescence from the GaAs grown by using this technique suggested that Ge acts as a successful buffer layer. M. Brongersma (Stanford) presented an overview of plasmonic research, an area that will take research into the realm of terahertz frequencies and nanoscale dimensions.

Symposium Support: Agilent Technologies, Univ. of Siegen-Ctr. of Micro- and Nanochemistry
$\mathcal{E}$ Engineering, Univ. of Virginia-Dept. of Electrical and Computer Engineering.

\section{Defect Engineering Matures to the Point of Fine-Tuning}

(See MRS Proceedings Volume 864)

Symposium E on Semiconductor Defect Engineering-Materials, Synthetic Structures, and Devices was part of a recurring series initiated at the 1992 MRS Spring Meeting. The entrenched maturity of the topic was reflected by the vast majority of presentations that dealt with the engineering aspects of defects, going beyond mere defect control and characterization.

The theoretical basis of doping in semiconductors was addressed in the opening invited talk by A. Zunger (NREL) as he explored doping strategies and guiding rules for hard-to-dope semiconductors. Other issues discussed included solubility rules, avoidance of "killer defects," and doping rules pertaining to local defect bonding effects (co-doping and cluster doping).

The use of impurities to control the morphological and thermal stability of NiSicurrently being developed for Si VLSIwas discussed by D.Z. Chi (IMRE, Singapore). He showed that the addition of a few percent of $\mathrm{Pt}$ to NiSi delays disilicide formation and also prevents agglomeration. $\mathrm{Ni}(\mathrm{Pt})$ offers work-function tuning in Si MOS over the range of $4.6-5.2 \mathrm{eV}$. Implantation of $\mathrm{F}$ and addition of $\mathrm{Ti}$ to $\mathrm{NiSi}$ were other examples of impurity engineering to control the properties of silicides. V. Moroz (Synopsys) described a technique for the fabrication of ultrashallow junctions for 65-nm-node Si technology. By carefully engineering the amorphization, point defects, and stresses induced by coimplantation of $\mathrm{Ge}, \mathrm{C}$, and $\mathrm{B}, \mathrm{Moroz}$ and collaborators were able to achieve high boron solubility along with the suppression of transient-enhanced diffusion.

It is now possible to control, with atomicscale precision, the position of individual dopant atoms at the semiconductor surface by using STM. This is important for the future fabrication of atomic-scale devices in silicon such as single-electron transistors or qubits in solid-state quantum computers. P.M. Voyles (Univ. of Wisconsin) reviewed his work on imaging individual dopant atoms using STEM. He showed that $Z$-contrast STEM can now image high- $Z$ impurities in low- $Z$ host crystals, one impurity at a time.

Light-induced degradation of amorphous Si solar cells is a well-known phenomenon. In contrast, the occurrence of a similar effect in crystalline Si cells has received scant attention in the past. J. Schmidt (Institute of Solar Energy Research, Hameln/ 
Emmerthal, Germany) reviewed this efficiency reduction in high-quality, singlecrystal CZ Si cells during (initial) illumination, and attributed it to the formation of $\mathrm{B}_{s} \mathrm{O}_{2 i}$ complexes in a recombination-assisted defect reaction process under illumination. It was pointed out that replacement of boron by gallium as acceptors in CZ-Si should suppress this problem.

Implantation of the light elements, $\mathrm{H}$ and $\mathrm{He}$, is known to create extended defects such as platelets and void regions in $\mathrm{Si}$, with applications such as wafer splitting and impurity gettering. E. Ntsoenzok (CNRS/CERI, Orleans, France) reported his recent landmark results on the use of rare-gas implantation ( $\mathrm{Xe}$ and $\mathrm{Kr}$ ) to generate bubbles and cavities in $\mathrm{SiO}_{2}$. This results in significant lowering of the dielectric constant of the $\mathrm{SiO}_{2}$ (from 3.9 to 1.5), opening exciting possibilities for the silicon oxide as a low- $\kappa$ dielectric. The Xe-implanted $\mathrm{SiO}_{2}$ layers show excellent mechanical resistance and high thermal stability.

Diluted nitride alloys currently attract considerable research interest due to their significant bandgap modulation. K.M. Yu (LBNL) talked about a new effect in these materials, namely, the mutual passivation of a dopant and an alloy element- $\mathrm{N}$ and $\mathrm{Si}$ in GaNAs:Si. This passivation is the result of $\mathrm{Si}(\mathrm{Ga})-\mathrm{N}(\mathrm{As})$ pair formation. It is thermally stable, contrary to the passivation of nitrogen by hydrogen in GaAsN. It should find uses in electrical isolation, bandgap engineering, and quantum confinement.

A very sensitive $3 \mathrm{D}$ hydrogen microscopy technique based on coincident elastic proton-proton scattering was described by G. Dollinger (Institut für Angewandte Physik und Messtechnik, Neubiberg, Germany). It is able to detect at a level lower than 1 atomic ppm with lateral and depth resolutions of better than $1 \mu \mathrm{m}$ and $5 \mu \mathrm{m}$, respectively. Using this microprobe, large amounts of hydrogen at the grain boundaries of polycrystalline diamond have been directly observed.

The symposium also held a tutorial on Semiconductor Heterojunctions: Properties and Photoelectronic Characterization, given by Y.N. Mohapatra (IIT, Kanpur).

Symposium Support: Eastman Kodak Co., Freescale Semiconductor, HORIBA Jobin Yvon, Intel Corp., JEOL SA, NREL, PARC, SAIREM, SOITEC PICOGIGA, Thales Research and Technology France, and Unisoku Co.

\section{Compound Semiconductor Thin Films Attractive for Practical PVs}

(See MRS Proceedings Volume 865)

Thin-film compound PVs have demonstrated energy conversion efficiencies approaching $20 \%$ and are leading candidates to provide lower cost solar energy due to potential advantages in manufacturing and materials costs. Symposium F on Thin Film Compound Semiconductor Photovoltaics focused on chalcogenide semiconductors such as $\mathrm{Cu}(\mathrm{InGa})(\mathrm{SeS})_{2}$, $\mathrm{CdTe}$, and CdS; on transparent conducting oxides; and on PV devices using these materials.

The application of recent advances in materials characterization to compound semiconductor thin films was a theme that carried over from the symposium tutorial to the technical sessions. I. Robertson (UIUC) reviewed the application of HRTEM-based characterization and showed that $\mathrm{CdS}$ grown on $\mathrm{Cu}(\mathrm{InGa}) \mathrm{Se}_{2}$ forms nanodomains and grows epitaxially on some $\mathrm{Cu}(\mathrm{InGa}) \mathrm{Se}_{2}$ grain surfaces. Several speakers presented results based on photoelectron spectroscopy (PES) of $\mathrm{Cu}(\mathrm{InGa}) \mathrm{Se}_{2}$ and $\mathrm{CdTe}$. R. Hunger (Darmstadt Univ. of Tech.) showed how angle-resolved PES has been used to measure valence-band states in $\mathrm{CuInSe}_{2}$ with good agreement to theoretical calculations. G. Teeter (NREL) used PES and other techniques to determine the kinetics of the $\mathrm{Cu}$ reaction with $\mathrm{CdTe}$. Spectroscopic ellipsometry was shown to be a valuable tool for optical characterization, with new results on off-stoichiometry of $\mathrm{Cu}(\mathrm{InGa}) \mathrm{Se}_{2}$ and the growth of oxides and CdTe.

Several speakers also presented results on new materials. T. Nakada (Aoyama Gakuin Univ.) showed that $\mathrm{Ag}(\mathrm{InGa}) \mathrm{Se}_{2}$ films can have either $p$ - or $n$-type conductivity, and solar cells with high open-circuit voltage were demonstrated.

The symposium included two discussion sessions. In the discussion on "Back Contacts: Are they the Key to Improved $\mathrm{Cu}(\mathrm{InGa}) \mathrm{Se}_{2}$ and CdTe Solar Cells?," there was general agreement that the contacts are controlled by interfacial layers, but the effects on film growth and devices are still poorly understood. The discussion on "Critical Issues for ThinFilm Polycrystalline PV: What Do We Have and Where Are We Going?" was dominated by efforts to define the relative value of fundamental and more applied research for helping the nascent PV industry.

Symposium Support: AFRL/VSSV, DuPont Central RED, NREL, and Shell Solar Industries.

\section{Gate Dielectrics Try to Stack Up to High-Mobility Semiconductor Substrates}

Symposium G brought together experts from semiconductor and metal oxide research communities to debate challenging problems of gate dielectric stacks on high-mobility semiconductor substrates for future generations of highperformance logic devices. H. Shang

\section{Inaugural "Science as Art" Competition Held at the 2005 MRS Spring Meeting}

As a special feature of the 2005 MRS Spring Meeting in San Francisco, a "Science as Art" competition was conducted. It complemented the Symposium X review presentations on materials used for flavors, fragrances, and art. It also coincided with the opening, in San Francisco, of an exhibition of sculpture highlighting the interplay between art and science.

The 1st place awards went to

J.W.P. Hsu, Sandia National Laboratories;

James C. Weaver, University of California, Santa Barbara; and

Thomas Sounart, Sandia National Laboratories.

The 2nd place awards went to

Frederick Sansoz, University of Vermont;

J.J. Penninkhof, California Institute of Technology;

Yihong Wu, National University of Singapore; and

Lim Wen Pei, National University of Singapore.

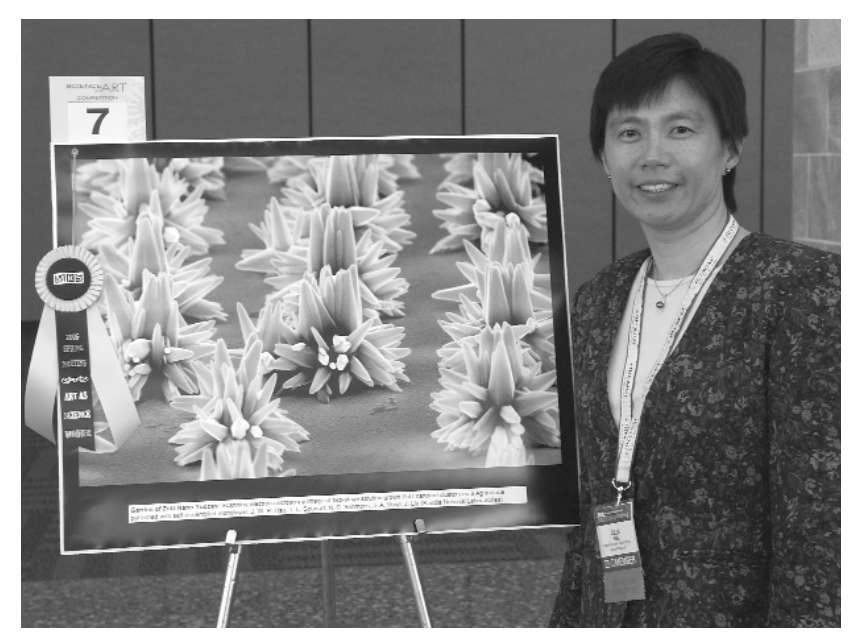


(IBM) showed enhanced channel mobility in strained Ge transistors, although the $\mathrm{SiO}_{2}$ gate dielectric used may not be the best solution for scalable devices. Despite significant progress, all projections of $\mathrm{Ge}$ MOSFET performance based on experimental results underperform state-of-theart Si devices, while N MOSFETs are barely functional. As Chi On Chui (Intel) said, dopant diffusion and activation deserve a closer look in order to understand the poor performance. M. Meuris

\section{How to Obtain Funding: From Research Needs to the Marketplace}

This year, the MRS Spring Meeting provided numerous opportunities for attendees to learn how to obtain funding, whether for research or business endeavors. Presentations were given by U.S. government agencies as well as a venture capitalist.

An interactive workshop, "The Road to Entrepreneurship," was designed for would-be entrepreneurs who are interested in learning about company formation and the venture capital fundraising process, as well as for materials researchers who are considering employment with venture-backed private companies. The presenter was Bill Frezza, general partner of Adams Capital Management Inc., a national venture capital firm that manages $\$ 700$ million and invests in first-stage semiconductor, information technology, telecommunications, and advanced materials companies. Frezza presented various ideas on goal assessment, performance metrics, commercialization timing and market readiness, market sizing and structure, team building, and business plan development. He provided valuable information on how best to obtain venture capital from investors like him. He fielded various questions from the audience.

Speakers from the Advanced Technology Program (ATP) of the National Institute of Standards and Technology (NIST) described how their program helps U.S. companies strengthen the integration of business and technical planning. Michael Shen, group leader in the ATP Information Technology and Electronics Office, placed the program within the context of a globally competitive world in which programs like ATP are forced to focus on the short-term returns on investments. ATP provides early-phase funding for companies. Frank Barros, business specialist in the ATP Information Technology and Electronics Office, gave practical information for submitting a proposal. The key question of funders, he said, is what the business potentials are of the technical innovation. Three panelists then spoke about their experiences with ATP funding and took questions from the audience. The panelists were Lisa Dhar of InPhase Technologies, Michael Natan of

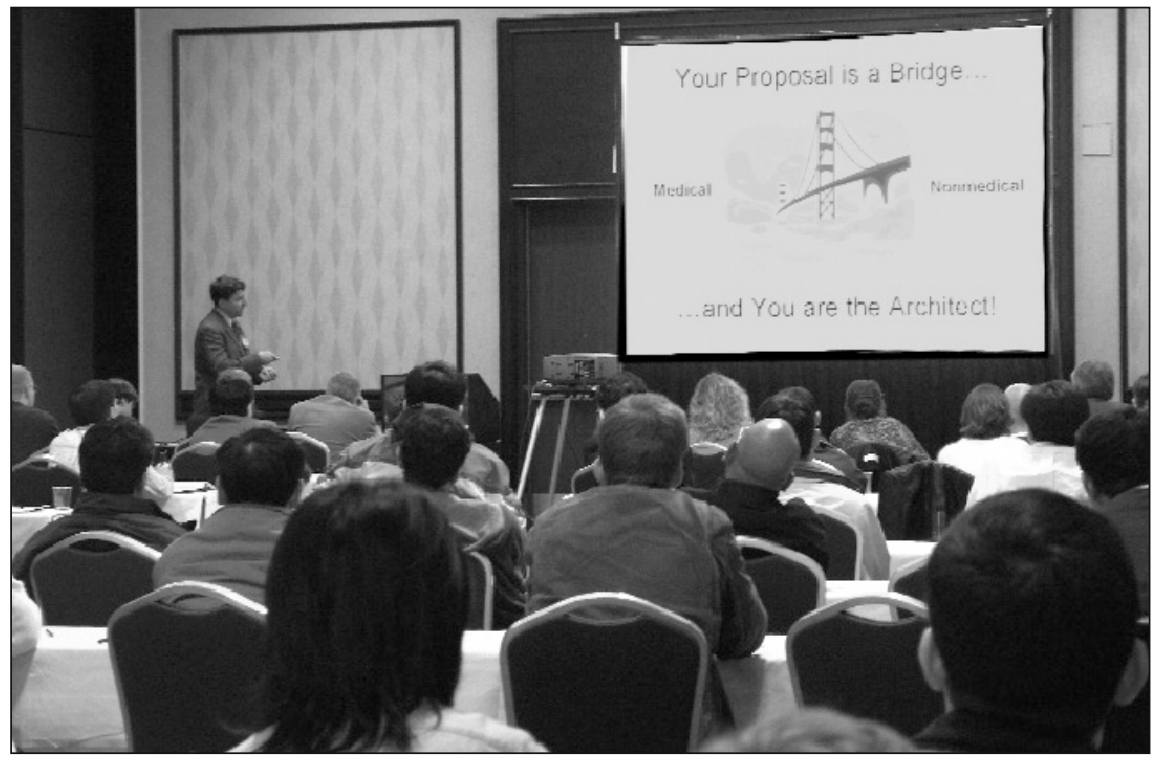

P.B. Messersmith of Northwestern gives practical information on how to write a proposal to receive research funding from $\mathrm{NIH}$.

Nanoplex Technologies, and David van Heerdon of Reactive Nanotechnologies. Dilip Banerjee, program manager in the ATP Chemistry and Life Sciences Office, moderated the panel discussion.

In a complimentary workshop held on the day of tutorials, speakers gave practical information on proposal writing for research funding from U.S. government agencies. The key message for attendees was twofold: to explore the institutes' Web sites for grant proposal guidelines and to discuss research ideas with a program director or manager before preparing and submitting the proposal. In this way, scientists can determine the best program within the institution where their research may get funded. A particu-

National Institutes of Health (NIH): Bioengineering Consortium (BECON) www.becon.nih.gov Center for Scientific Review www.csr.nih.gov

National Science Foundation (NSF) www.nsf.org lar challenge in preparing a proposal for the National Institutes of Health $(\mathrm{NIH})$ resides in making the connection between materials research and the medical field. Materials researchers were highly advised to obtain help from consultants and find collaborators to supplement the materials expertise with the medical. For the National Science Foundation (NSF), proposals must provide a strong educational component along with the materials research idea.

W. Lance Haworth, executive officer of the Division of Materials Research, described the proposal process at NSF. John Bowers of the Center for Scientific Review provided an overview of the grant proposal process at NIH, using nanoscience and biomaterials topics as supported by BECON as an example. BECON is the NIH Bioengineering Consortium. Phillip B. Messersmith, associate professor at Northwestern University, followed with a presentation from his perspective as a principal investigator or co-investigator who has received nine NIH grants. Messersmith is a permanent member of the Biomaterials and Biointerfaces Study Section at NIH. 
(IMEC) and others also stressed the fact that a high density of interface defects in the upper half of the energy bandgap may be part of the N MOSFET problem.

It becomes increasingly clear that the passivation of Ge or III-V compound semiconductor surfaces is the most important (but also challenging) task. K. Saraswat (Stanford) reviewed surface passivation of Ge by GeON, and K. Kim (Harvard) showed that ALD-grown hafnium nitride was a superior passivating layer for Ge, while S. Rivillon (Rutgers) presented new results demonstrating that hydrogen passivation of the Ge surface was possible. D.-L. Kwong (UT-Austin) showed that surface treatment by silane and phosphine produced the best Ge MOS capacitors and transistors with $\mathrm{HfO}_{2}$ gates.

A number of presentations addressed metal gates as well as the growth and physical and electrical characterization of high-к (complex) oxides on silicon substrates. J. Robertson (Cambridge) explained why the implementation of dual metal/high- $\kappa$ gates with the required (low) threshold voltages was possible despite the fact that the band-alignment pinning factor for high-к oxides was less than unity. The metal gate solution becomes increasingly important for highperformance devices, as poly-Si gates do not seem to satisfy device requirements due to problems with scaling and control of the threshold voltage $V_{t}$ (especially for $P$ MOSFET devices). Several papers proposed metal gate options, including metal bilayers and fully silicided gates. At the same time, materials-related issues such as interactions with gate dielectrics, interdiffusion, and oxygen diffusion were covered. J.-P. Locquet (IBM-Zurich) and L. Pantisano (IMEC) demonstrated that $\mathrm{HfO}_{2}$ and $\mathrm{La}_{2} \mathrm{Hf}_{2} \mathrm{O}_{7}$ layers fabricated by MBE could be successfully integrated with a pilot $200 \mathrm{~mm}$ processing line to fabricate fully functional short-channel $(100 \mathrm{~nm})$ MOSFETs with performance comparable to or better than ALD-made devices.

Symposium Support: Applied Materials, European Commission 6th Framework Programme, Freescale Semiconductor, Genus, IBM T.J. Watson Research Ctr., and Umicore.

\section{Back to Big: Giant-Area Electronics Spreads Its Reach}

(See MRS Proceedings Volume 870E)

Advances in the development of giantarea ICs on flexible substrates are expected to lead to applications ranging from PV devices and flexible displays to "sensitive skin" for robotics and medical applications. Recent developments in this area were covered in Symposium $\mathrm{H}$.

J. Yang (United Solar) presented an overview of the thin-film solar cell indus- try. The industry is now using giant nonconventional substrates, such as milelong stainless steel foils, in a roll-to-roll deposition process to make amorphous silicon solar cells. It might serve as a model that other applications in giantarea electronics will follow. The expected worldwide production volume for solar cells of $15 \mathrm{GW}$ installed power per year in 2020 translates into a substrate area of about $10 \mathrm{~km} \times 10 \mathrm{~km}$ covered with costeffective and highly efficient solar cells.

M. Schubert (Univ. of Stuttgart) reported on recent progress toward wearable PVs and assessed the prospects for crystalline- and amorphous-based solar cells for this application. His group has recently demonstrated 5\% efficiency in silicon solar cells fabricated at $110^{\circ} \mathrm{C}$ that are compatible with textiles. This technology is very close to commercialization. M. Hack (Universal Display Corp.) described the development of OLED fabrication on steel foil, reporting on OLEDs yielding up to $37 \mathrm{Cd} / \mathrm{A}$ with lifetimes of up to $100,000 \mathrm{~h}$ for commercial red and green devices, and yielding up to $8 \mathrm{Cd} / \mathrm{A}$ for the blue OLEDs still under development.

A recurring symposium theme was new and cheaper methods for patterning largearea displays. Also, methods to construct solid-state lighting panels, which can be directly plugged into $110 \mathrm{~V}$ mains and are fault-tolerant, were discussed.

S. Wagner (Princeton) said that the large-area electronics field is in the same growth mode as the more conventional microelectronics industry was in the late 1980s. He predicted that giant-area electronics will have a very large penetration into the consumer market because TFT circuits are estimated to be much cheaper than conventional crystalline silicon. The challenges include the development of novel electronic devices, which can be mechanically stretched, bent, or molded onto complicated-shape surfaces. According to Wagner, these advances will involve many new and unforeseeable materials issues, making the field of giant-area electronics "experimenter's paradise" for many years to come.

S. Mohapatra (Organic ID, Austin) reported on organic TFT rectifiers working at $15 \mathrm{MHz}$ or higher. These circuits are important building blocks for future organic RFID tags. V. Chu (INESC MN, Portugal) analyzed new techniques to functionalize semiconductor devices with DNA using voltage pulses and novel optical methods. These techniques have allowed her to detect DNA using integrated thin-film photodetectors.

The symposium participants discussed and addressed the many-faceted and multidisciplinary challenges that have to be overcome during this walk through "experimenter's paradise."

\section{Organic Semiconductors Feed into Thin-Film Electronics}

(See MRS Proceedings Volume 871E)

Symposium I on Organic Thin-Film Electronics brought together researchers engaged in science related to the synthesis of new organic semiconducting materials, the fabrication of devices, and the mechanisms underlying device operation. The tutorial given by N. Tessler (Technion, Israel) and D. MacKenzie (Kovio Inc.) opened the discussion on the basic processes governing the operation of organic thin-film devices and the challenges to create new functionality and low-cost products using these organicbased devices. Throughout the technical sessions, the synthesis of new materials, charge transport mechanisms, charge injection, and recombination were discussed in the context of devices including FETs, LEDs, memory, and PV devices.

I. McCulloch (Merck, U.K.) reported on the synthesis of terthiophene polymers that exhibit good environmental stability and field-effect mobilities of $0.6 \mathrm{~cm}^{2} / \mathrm{V} \mathrm{s}$. $\mathrm{S}$. Jenekhe (Univ. of Washington) presented results on a new $n$-type oligoquinolinebased material used as an emissive and electron transport material for blue OLEDs, and M. Galvin (Univ. of Delaware) discussed the structure/property relationships that govern the performance of polymerbased devices. M. Turner (Manchester) gave an overview of the work done by the Engineering and Physical Sciences Research Council consortium in organic electronics in the United Kingdom and D. Cupertino (Avecia, U.K.) discussed the device stability of polytriarylaminebased TFTs.

Studying electrical stability and charge transport is extremely important for practical applications such as display backplanes and RFID tags. A. Salleo (PARC) showed that the field-effect mobility of polythiophene-based TFTs is limited by charge trapping at the channel and related the trap populations to operating voltages and device lifetime. D. Frisbie (Univ. of Minnesota) presented work on the development of crystalline organic semiconductors that show improved $n$-channel properties. A. Kahn (Princeton) discussed the role of $n$ - and $p$-type electrical doping in organic thin films.

The understanding of organic-based devices, along with the development of new materials, has led to a wide variety of applications in the field. G. Jabbour (Arizona State Univ.) gave an overview 
of the different concepts and structures used to achieve memory devices for data storage applications and showed the memory effect on LED structures studied in his group; A.J. Epstein (OSU) discussed the potential for organic-based materials in spintronic devices. The use of organic materials in PV devices was also discussed. R.H. Friend (Cambridge) presented work on the development of polymer brushes as a matrix for nanocrystals, resulting in a charge collection efficiency of $80 \%$. D. Ginley (NREL) used nanostructured materials, in the form of nanoparticulate materials blended with polymers or surface-bound organized nanostructures, to obtain efficiencies of $0.5 \%$ and currents of up to $3.5 \mathrm{~mA} / \mathrm{cm}^{2}$. P.W.M. Blom (Univ. of Groningen, the Netherlands) demonstrated that the photocurrent of solar cells based on blends of polymer and [6,6]-phenyl C61-butyric acid methyl ester is limited by the slowest charge carrier species and discussed the role of blend composition on $e^{--h}$ pair generation and light absorption. During the Meeting, there were demonstrations of practical applications such as OLED displays by M. Kreger (Add-Vision), printed backplanes for displays by $\mathrm{C}$. Newsome (Epson, U.K.), and electronic circuits for tagging by Z. Dietmar (PolyIC, Germany).

Symposium Support: Ciba Specialty/CSEM SA, Covion Organic Semiconductors, Merck Chemicals UK, Organic ID, PARC, Plastic Logic, and Universal Display Corp.

\section{Synergy between Experiments and Simulations Emphasized in the Study of Structure and Mechanical Behavior of Nanophase Materials \\ (See MRS Proceedings Volume 872)}

The overall aim of Symposium J, Micro- and Nanosystems-Materials and Devices, was to bring together scientists working in various aspects of the development of new materials and devices for micro- and nanotechnologies, as most of the projects are multidisciplinary. A wellattended tutorial on bioMEMS was presented by R. Bashir (Purdue), K. Turner (MIT), and D.A. LaVan (Yale).

Invited speakers included J. Michael (SNL), who reported on work combining FIB and EBSD to characterize MEMS materials and devices; J. Kelly (SNLLivermore), who reported on highaspect-ratio electrodeposition using the LIGA process, and R. Maboudian (UC-Berkeley) who addressed surface and materials issues for MEMS. Contributed talks and posters spanned many topics, including device characterization, fabrication, properties, microstructures, modeling, and applications.

\section{A Playground of Structures Gains Inspiration from Biology}

(See MRS Proceedings Volume 873E)

Symposium $\mathrm{K}$ on biological and bioinspired materials was striking in its diversity, but perhaps more striking in its unanimity. Amazed at nature's fascinating structures and functional materials, researchers were inspired by one another's attempts to understand and recreate some of these biological solutions to materials challenges.

This is an exciting time for diatom research, since every stage of biosilica formation, which is "engineered" by singlecelled algae called diatoms, is under scrutiny. This past year has seen the first publication of a complete diatom genome. Invited speakers presented the unique proteins related to silicification that have been identified and their complementary roles in silica nucleation and regulation. In vitro studies on synthetic macromolecules demonstrated a refined understanding of the important properties of these proteins, marking a key advance for biomimetic processing. The session was rounded out with presentations of diatom-inspired materials synthesis, including physical templating, the incorporation of germanium along with silicon into diatoms, and chemical transformations of silica structure into industrial oxide materials such as barium titanate.

Subsequent sessions, including two nights of posters, delved into issues of molecular and mineral assembly in more detail. Methods were shown to assemble DNA molecules into specific structures. Polymer loops were modeled to gain an understanding of muscle tissue. The assembly of protein cages was discussed; in particular, ferritin cages may be important not only for iron storage within an organism but also as nanoreactors to confine the formation of industrial nanoparticles. Analogous work was presented for 2D organic films: by engineering selfassembled monolayers, it was shown how specifically oriented mineral crystals can form. These presentations emphasized that macromolecular assembly on a variety of length scales can contribute to the structures of useful composite materialsor even machines, including ATP-driven nanomotors and other futuristic-seeming achievements, which were demonstrated.

Speakers illuminated current ideas in animal optics, covering newly discovered optical properties of biomaterials, the strange visual worlds of marine organisms, and implications for evolution. Likewise, in a joint session with Symposium $\mathrm{L}$ on the mechanical properties of biomaterials, speakers introduced work on bone and other structural biomaterials. The topic of bone was taken up a second time in the final sessions of the symposium, where speakers concentrated on achieving a deeper physical understanding of the biochemistry of mineralization. In situ AFM and synchrotron x-ray scattering and spectroscopy were emphasized. In the final talks, research aimed specifically at biomedical implants was presented. Attendees challenged each other on a wide array of approaches to bone implant materials, including titanium, graded polymer composites, and calcium phosphate coating methods.

Symposium Support: NIH-NIDCR.

\section{Materials Science Spurs Understanding of Biology}

There is an increasing awareness among basic and clinical researchers that an understanding of the structure and mechanical properties of natural biological materials will have a significant impact on the future of biology and biomedicine. These two fields are recognized as areas that will undoubtedly gain in importance in a progressively aging population as exemplified by the United States and other countries. The very point was noted emphatically by George M. Whitesides (Harvard) in his MRS plenary address, with the additional comment that materials scientists in particular could contribute immensely to progress in biological and biomedical endeavors. The emergence of structural and mechanical analyses in biomineralization, biomimetic processing, self-assembly, biomaterials development, and microfabrication, for example, should have considerable effects on the means by which health care is improved for the general public worldwide.

With this backdrop, Symposium L presented the theme of Structure and Mechanical Behavior of Biological Materials. The symposium, separately and in joint session with Symposia K and BB, examined aspects of the most recent knowledge of the mechanical properties of hard and soft biological tissues and the current state of functional biomaterials and biomimetics, as well as nanostructured biomaterials. With respect to mechanical properties, R. Richie (UC-Berkeley/LBNL) described the fracture, fatigue, and aging of bone and dentin as examples of hard mineralized tissues undergoing natural changes in structure and mechanical behavior, and P. Thurner (UCSB) and M. Morris (Univ. of Michigan) reported, respectively, the responses of bone to high local strains and mechanical loading. J. Freeman (Univ. of Virginia) studied elastic energy storage in collagen found in tendons and suggested 
a future direction for tissue-engineered collagenous materials utilizing the principle of energy storage. P. Zaslansky (Weizmann Inst.) demonstrated the novel analytic approach of speckle interferometry to assess mechanical changes in mineralized biological specimens.

Functional biomaterials and biomimetics focused on bone and dentin but also included reports concerning invertebrate structures. S. Weiner (Weizmann Inst.) provided insights into lamellar bone structure and function, and A. Evans (UCSB) addressed mechanisms underlying the inelastic deformation of bone. P. Hansma (UCSB) proposed a mechanical role in bone for certain organic molecules whose loss in the tissue would possibly lead to debilitating osteoporosis or other bone diseases in humans. J. Aizenberg (Bell Labs/Lucent) presented an overview of startling results demonstrating a highefficiency fiber-optical network in a deepwater glass sponge, and R. Naik (AFRL) detailed biomimetic materials chemistry.

Nanostructured biomaterials were investigated through studies of stiffening in biopolymers, as reported by E. Van der Giessen (Univ. of Groningen), and in a related presentation, D. Raabe (MPI) gave details on texture and smart anisotropy in nanocomposites. C. Ortiz (MIT) described her interesting and precise work concerning the nanomechanics of cartilage aggre- can molecules utilizing functionalized tips for AFM.

A further discussion of soft biological tissues and their mechanical properties was led by X. Li (Univ. of South Carolina), who presented AFM imaging and nanomechanical testing of a variety of cells and tissues. L. Gibson (MIT) described fibroblast contraction of collagen glycosaminoglycan scaffolds, and U. Schwarz (MPI) demonstrated how mechanosensing influences cell organization. Additional hard-tissue studies were given by A. Choo (Univ. of British Columbia), in his talk on spinalcord injury related to injury mechanism; B. Pokroy (Technion, Haifa), in his discussion of potential organic molecule

\section{Poster Prizes Awarded at the 2005 MRS Spring Meeting}

The 2005 MRS Spring Meeting chairs awarded prizes for the best poster presentations. Prize recipients received $\$ 500$, a certificate, and the honor of having their posters on display for the remainder of the Meeting. Posters and authors awarded prizes were (A9.9) Effect of PostOxidation of Silicon Nanocrystals as a Floating Gate of Nonvolatile Memory, J.M. Kim, M.K. Choi, E.K. Lee, C.J. Kang, and Y.S. Kim (Myongji University, South Korea); and Y. Khang, E.H. Lee, K.Y. Lee, and J.H. Lee, (Samsung Advanced Institute of Technology, South Korea); (F14.25) High-Efficient ZnO/PVD$\mathrm{CdS} / \mathrm{CuIn} \mathrm{Ga}_{1-x} \mathrm{Se}_{2}$ Thin-Film Solar Cells: Formation of the Buffer-Absorber Interface and Transport Properties, M. Rusu, C.A. Kaufmann, A. Neisser, S. Siebentritt, Th. Glatzel, and M.Ch. LuxSteiner (Hahn-Meitner Institute, Berlin); (I3.9) Low-Voltage All-Polymer Transistor Utilizing a Hygroscopic Insulator, T.G. Backlund, R. Osterbacka, and H. Stubb (Abo Akademi, Finland); and H.G.O. Sandberg (Abo Akademi/VTT Information Technology, Finland); (I6.18) Conjugated Oligoquinolines: $n$-Type Organic Semiconductors for Highly Efficient Blue Electroluminescence, A.P. Kulkarni, A.P. Gifford, C.J. Tonzola, and S.A. Jenekhe (University of Washington, Seattle); (K9.21) Fabrication and Characterization of Active Matrix Array for Cell Probing and Screening, S.-I. Jun and P.D. Rack (University of Tennessee), T.E. McKnight (Oak Ridge National Laboratory), and A.V. Melechko and M.L. Simpson (University of Tennessee/Oak Ridge National Laboratory); (L5.9) Probing Electromechanical Properties of Biological Systems Down to the Nanoscale, A. Gruverman and B.J. Rodriguez (North Carolina State

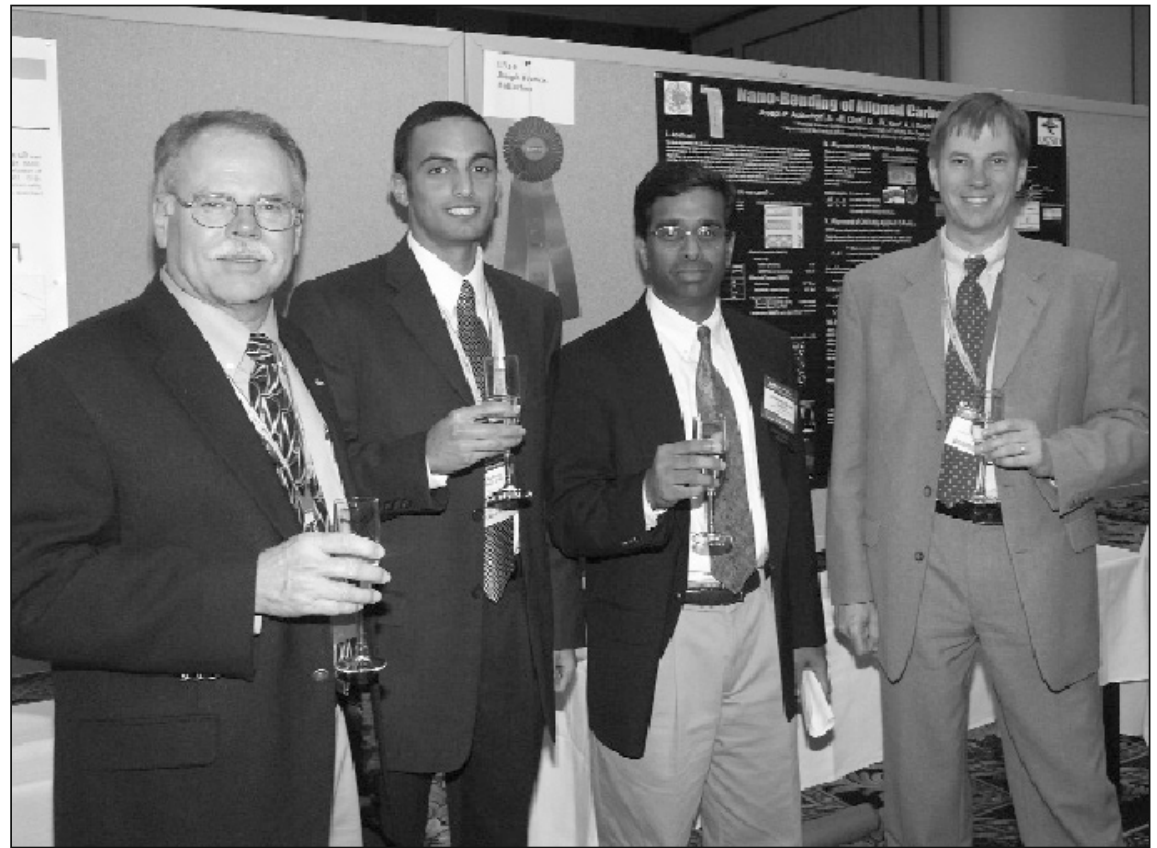

University), and S.V. Kalinin (Oak Ridge National Laboratory); (O12.15) Influence of Stress on Structural and Dielectric Anomaly of $\mathrm{Bi}_{2}\left(\mathrm{Zn}_{1 / 3} \mathrm{Ta}_{2 / 3}\right)_{2} \mathrm{O}_{7}$ Thin Film, J.H. Noh, H.B. Hong, and K.S. Hong (Seoul National University); (R8.60) Mechanistic Studies of the Formation of Alumina SolGels by Multinuclear NMR, S.C. Chinn, A.M. Sawvel, T.F. Baumann, J.H. Satcher, and R.S. Maxwell (Lawrence Livermore National Laboratory); (S6.30) DNA Separation Using Gold/Magnetic Iron-Oxide Composite Nanoparticles, T. Kinoshita, S. Seino, Y. Otome, T. Nakagawa, T. Nakayama, T. Sekino, K. Niihara, and T.A. Yamamoto (Osaka University), and Y. Mizukoshi (Osaka Prefectural College of Technology); (U3.10) Nano-Bending of Aligned Carbon Nanotubes, J.F. AuBuchon, L.-H. Chen, D.-W. Kim, A. Gapin, C. Daraio, and S. Jin (University of California, San Diego); (Z3.24) Morphology-Controlled Synthesis of Platinum Nanostructures, J. Chen, T. Herricks, M. Geissler, and Y. Xia (University of Washington, Seattle); and (Z10.12) Synthesis and Applications of the Hollow Nanocrystals, Y. Yin (Lawrence Berkeley National Laboratory), A. Cabot (University of California, Berkeley), and C. Erdonmez, J.A. Shaw, and A.P. Alivisatos (University of California, Berkeley/Lawrence Berkeley National Laboratory). 


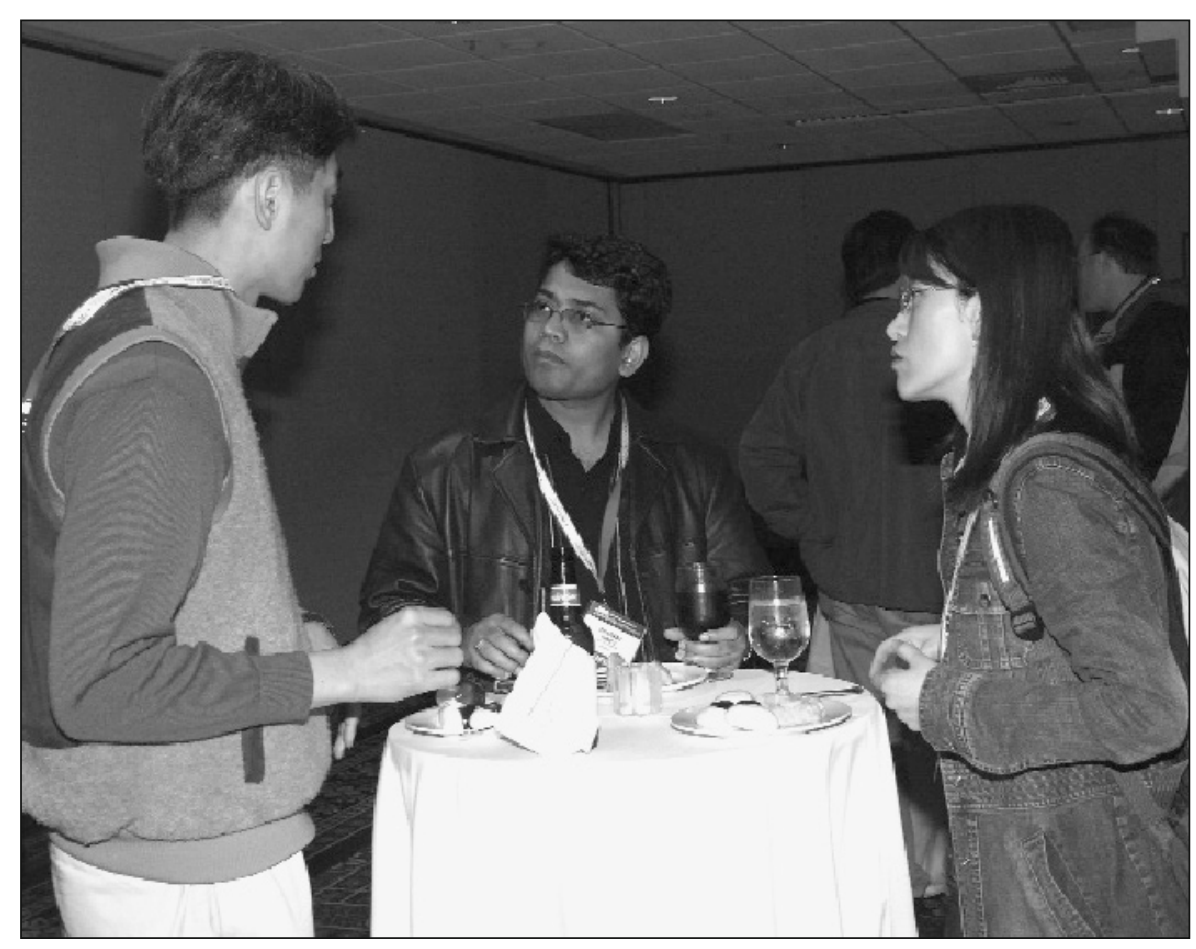

Students enjoy refreshments and conversation at the student mixer.

mediation in biogenic aragonite compared with geological aragonite; J. Arias (Universidad de Chile), in his work characterizing the microstructure of the barnacle shell; E. Donnelly (Cornell), in her investigations of collagen content and organization related to bone nanomechanical properties; and A. Woesz (MPI), in his examination of bone replacement materials with designed architecture.

\section{Bioadhesion, Supramolecular Assemblies Examined at Nano-Bio Interfaces}

The development and control of nanoscale interfaces for integrating natural and synthetic materials was the focus of Symposium M, Developing Nano-Bio Interfaces. The control of interfaces involving proteins, membranes, or cells was the focus of several presentations and posters. D. Leckband (UIUC) kicked off the symposium by exploring principles underlying biological adhesion, stressing that the understanding of molecular mechanisms is critical to the elucidation of cell adhesion. Another highlight was a presentation by J. Groves (UC-Berkeley) on the dynamic control of the interface between $T$ cells and artificial, patterned substrates functionalized with specific antigens. A. Chilkoti (Duke Univ.) addressed the state of the art in dip-pen nanolithography. He further described the application of enzymes teth- ered to the tip of an atomic force microscope for the fabrication of nanoscale structures, demonstrating the convergence of biology and nanotechnology. J. Schmidt (UCLA) spoke on synthesizing triblock copolymer membranes having increased stability and an ability to mimic lipid bilayer structures, including maintaining the functionality of inserted membrane proteins. A. Parikh (UC-Davis) outlined a number of methods for patterning supported lipid bilayers with $100 \mathrm{~nm}$ resolution and also presented methods of interrogating the properties of these patterned membranes. Other related topics included the development of nanostructured interfaces and their influence on protein and cell adhesion and properties. For example, J. Liu (SNL) described an electrochemical method relying on nanofabricated surfaces to achieve attomolar sensitivities, a dramatic improvement over traditional electrochemical methods.

Another series of presentations considered the themes of supramolecular assemblies and bionanodevices. K. Keren (Stanford) and H. Yan (Arizona State Univ.) spoke on using DNA as a biomolecular template for organizing nanomaterials. Keren presented the use of DNA to attach carbon nanotubes and form FETs by metallizing the DNA adjacent to the carbon nanotube, and Yan presented the ability to assemble DNA lattices, including switchable DNA lattices, useful for fabricating nanomaterials. Other talks focused on exploiting dynamic biomolecular processes for useful work. A. Mansson (Univ. of Kalmar) discussed the actinomyosin transport system, with an emphasis on developing multidimensional architectures that mimic the assembly of muscle fibers. Surface chemistry was shown to have a dramatic effect on the speed of myosin transport, and surface topography was shown to guide actin filament transport.

A final general topic was the development of biological and bio-inspired materials synthesis and assembly. Two talks focused on using icosahedral viruses and chaperonins for precision localization of nanoparticles. In both cases, genetic engineering was used to mutate the templates and impart selective adsorption of metallic and semiconductor nanoparticles. In the case of the icosahedral virus, B. Ratna (NRL) presented work on assembling conductive networks on the coat protein of the virus using gold nanoparticles assembled on the vertices of the virus shell. C. Paavola (NASA-Ames) showed work on assembling 2D lattices of metallic and semiconductor nanoparticles using the self-assembly of chaperonins. The ability to create functional hybrid assemblies was also highlighted in several talks, including one by $\mathrm{H}$. Baca (Univ. of New Mexico), who discussed the use of yeast cells to influence the phase and assembly of silica.

Symposium Support: AFOSR and Cytoskeleton.

\section{Influence of Polymer Gels Ranges from IR Sensing to Stem Cell Differentiation}

Symposium N on Polymer Gels for Emerging Technologies brought together scientists working in different areas of soft polymer gels. While the primary focus was on synthetic gels, J.-F. Joanny (Institut Curie) began with a theoretical discussion of active polymer gels that mediate cell motion. This biological theme continued with a variety of talks directed at biomedical applications of synthetic polymer gels. Examples included silicone gels for intraocular lenses with optical properties that can be adjusted noninvasively after surgical implantation (J. Kornfield, Caltech), adhesive hydrogels that mimic the performance of naturally occurring mussel adhesive proteins (P. Messersmith, Northwestern), and hydrogels designed to control the differentiation of stem cells (K. Anseth, Univ. of Colorado). In addition, several talks (D. Pochan, Univ. of Delaware; T. Deming, UCLA; and K. Kiick, Univ. of Delaware) described polypeptide- 


\section{Symposium X Covers Research, Nano Centers, and the Intersection of Art and Science, Materials and Biology}

Speakers in Symposium $X$ presented overviews of their fields, covering a blend of science and art, flavors and fragrances, biomineralization, silicon sculpturing, and correlated electrons, as well as a special feature on nanoscience centers at U.S. national laboratories.

In his presentation on "The Magic World of Flavors and Fragrances," Cyril Mahaim (Firmenich SA, Switzerland) said that flavors and fragrances act in a very primitive level in the human brain, eliciting emotional reactions. Although extensive research has been conducted, a lot of details remain unknown, including a thorough analysis of smells and flavors. Although chemicals can be analyzed with continually greater accuracy and greater sensitivity, smell can still not be described by an analytical measure, and some smells are detected by the human nose at doses where the most sophisticated instruments fail. Flavor, Mahaim said, is a complex combination of smell, taste, and "mouthfeel" (i.e., fat, temperature, and texture). In the $\$ 11$ billion business of flavors and fragrances (in which Firmenich is the third leader, with a $\$ 2$ billion share), the application of taste and smell in consumer products is very diverse, from laundry powders to luxury perfumes, from very simple natural citrus essential oils to complex meat flavors encapsulated to ensure the proper delivery in a difficult food application. In the coming decades, the industry will face three major challenges: delivery systems, new fragrances (as the palette is already well furnished), and regulations (as the pressure is increasing to fulfill the requirement of biodegradable materials in all applications).

Art and science can indeed work in tandem, representing true interdisciplinarity. This was evident in the second Symposium $X$ talk, which was given in a joint presentation by Rolf Gotthardt (EPFL, Switzerland), a physicist and electron microscopist, and Etienne Krähenbühl, a sculptor. Gotthardt gave the scientific explanation for shape-memory alloys (SMAs), while Krähenbühl described, in French accompanied by an interpreter, why and how he uses SMA in his sculptures.

Krähenbühl said, "Although I had been working in metal for many years, these alloys sparked new ideas, and my work completely changed, embracing lightness, movement, and memory. This exacting and rigorous world of science is at the same time playful and magical, and now it permeates all that I create."

In describing his recent research, Gotthardt said that his team is working toward influencing the shape-memory effect locally in thin films by ion irradiation, for use in fabricating micro-grippers, and is using electron microscopy to optimize the irradiated SMA microstructure. Gotthardt and Krähenbühl envision using an extremely thin SMA film in sculpture. They also plan to use the high damping capacity of SMAs to explore some temperature-induced sound effects in sculptures. Gotthardt demonstrated the effect by first banging on a bell which produced only a dull sound. However, after he immersed the bell in hot water, it produced a clear metallic sound.

In a presentation on biomineralization, Steve Weiner (Weizmann Inst., Israel) described a world in which organisms are able to control in remarkable ways the manner in which minerals form. One of the most common demonstrations of control is over the type of calcium carbonate polymorph formed. Organisms can do this with absolute fidelity, despite the fact that, crystallographically, some of them are very similar. Organisms can also shape crystals seemingly at will. One strategy for doing this, which has been identified in the past six years, involves taking advantage of disorder by first forming a highly metastable disordered phase, shaping it, and then crystallizing it. One such phase used by various invertebrate phyla is amor- phous calcium carbonate. Weiner said that this strategy has already proven useful for growing large, single, flat crystals of calcite, and it may have many applications in materials science.

Ulrich Goesele (MPI, Halle) gave an overview, partly in a historical sequence, on the development of nanostructured $\mathrm{Si}$, including nanoelectronics, nanoporous $\mathrm{Si}, \mathrm{Si}$ nanocrystals, and $\mathrm{Si}$ nanowires. He detailed some of his own work relating to $\mathrm{Si}$ quantum wires and porous Si formation in the early 1990s. He described the obvious versatility of $\mathrm{Si}$ on the nanoscale and its various useful properties, for use as photonic crystals and photonic structures, for example. He also presented data that indicates that $2-5 \mathrm{~nm}$ Si nanoparticles may be present in galactic dust. He said Si has clearly shown potential for the creation of nanoscale structures with a wide variety of possible variations.

Yoshinori Tokura (Univ. of Tokyo) gave an overview on recent progress made in oxide bulk/interface engineering. $\mathrm{He}$ proposed an emergent correlated electron technology. An electron in the periodic crystalline lattice behaves also as a wave, as does any other quantum particle. When many electrons with a comparable number of atoms are packed in a crystal, and the electron-electron repulsion interaction is strong enough, electrons are almost localized on the respective atomic sites or only barely mobile. Due to this revived particle nature, an electron in such a solid has three attributes; charge $(-e)$, spin $(S=1 / 2)$, and orbital. An orbital, which represents the electron's probabilitydensity distribution, can be imagined as the shape of an electron cloud in a solid. The charge, spin, and orbital degrees of freedom-and their coupled dynamics-can produce complex phases such as liquid-like, crystal-like, and liquid-crystal-like states of electrons, and phenomena such as electronic phase separation or nanometric pattern formation. The electronic and magnetic phases in the correlated electron systems, typically in transition-metal oxides, can be controlled in unconventional ways, in some cases with ultrafast response times. Tokura said that such newly won control offers hope that correlated electron systems may provide a basis for novel electronics. The colossal magnetoresistance effect in the manganese oxides is one such example, but the similar metal-insulator transition can be achieved by electric current injection, as anticipated in the application of new memory devices.

Julia Phillips of Sandia National Laboratories gave an overview of nanoscience research at the national laboratories in the United States. A major portion of the Department of Energy's participation is through five Nanoscale Science Research Centers, located at national laboratories around the country. These facilities are currently under construction at Oak Ridge, Los Alamos and Sandia, Lawrence Berkeley, Argonne, and Brookhaven. The centers, which will commence full operation over the next two years, are research facilities for the synthesis, processing, and fabrication of nanoscale materials. Each center is co-located with existing user facilities for characterization and analysis (e.g., synchrotron light sources, neutron-scattering centers, and microfabrication capabilities) and is designed to provide specialized equipment and support staff for nanoscience research not readily available to the research community. Each center will be operated as a user facility, available to all researchers worldwide based on proposals peer-reviewed for scientific merit. Even though the centers are still under construction, they all have a vibrant "jumpstart" user program that has been in place for several years to enable the scientific community to begin to access the unique nanoscience capabilities and expertise that are already resident in the laboratories. 
based or polysaccharide-based hydrogels with structural features designed to mimic naturally occurring gels.

A second major theme of the symposium involved the use of polymer gels in device applications, ranging from microfluidic devices (J. Moorthy, Univ. of Wisconsin) to infrared sensors (L. Brott, AFRL). U. Wiesner (Cornell) described a method for using volume phase transitions of polymer hydrogels to direct hydrogel motion, and G. Payne (Univ. of Maryland) described an electrodeposition process for patterning thin hydrogel layers.

The symposium concluded with a session on mechanical properties, with applications directed at both biological and device applications. J.P. Gong (Hokkaido Univ.) described a set of remarkably tough hydrogels with a double network structure, and elucidated the molecular-level structural requirements for the enhanced toughness. H. Hui (Cornell) and his collaborators described a mechanical test that can be used to measure the permeability of polymer hydrogels, and the symposium concluded with a set of talks on the mechanical deformation of polymer gels.

Symposium Support: SNL.

\section{Mechanical Properties of Nanostructured Materials and Polymers Considered}

\section{(See MRS Proceedings Volume 875)}

In Symposium O on Thin-Film Stresses and Mechanical Properties, speakers covered a wide array of studies on testing and characterization techniques, growth, structure, and plasticity of thin films. Of particular interest at this Meeting was an emphasis on the plasticity effects in nanostructured thin films and small structures. In a joint session with Symposium BB (Mechanical Properties of Nanostructured Materials-Experiments and Modeling), recent results from multiple groups using novel in situ testing techniques discussed observations of grain growth and rotation found in nanograined metallic thin films. Using a variety of experimental techniques, from in situ TEM to in situ synchrotron diffraction, researchers reported common observations of grain growth and possibly rotation in nanograined metallic films. Another notable trend across multiple sessions was the large amount of research being conducted on the mechanical properties of polymer films.

Symposium Support: Hysitron and WITec $\mathrm{GmbH}$.

\section{Study of Oxidation, Catalyst Dynamics Approaches "Real" Conditions}

Presentations in Symposium $\mathrm{P}$ gave a fascinating glimpse into the state of the art in understanding the dynamics of surface reactions using a wide variety of experimental techniques. The aim of the symposium was to study the science of gas-surface reactions under conditions ranging from UHV to high-pressure reactive environments, and many of the studies were carried out under environments close to those found in industrial applications.

Highlights of the symposium included detailed studies of the oxidation of different materials. In the semiconductor field, silicon oxidation is an essential step in device processing, and in situ synchrotron experiments probe the interfacial reaction in detail (A. Yoshigoe, SPring-8, Japan). Copper oxidation is a similarly important corrosion process. In situ UHV-TEM has been used to investigate $\mathrm{Cu}$ and $\mathrm{CuAu}$ oxidation, characterizing the effect of orientation, alloying, and surface structure on the oxide structure and growth kinetics (G. Zhu, ANL). In situ synchrotron studies provide complementary insight into this process (P. Fuoss, ANL) and are also important in determining growth stress due to high-temperature oxidation of alloys (B. Veal, ANL). It is important to determine growth stress, since this stress causes spallation of the high-temperature coating, including the thermal-barrier coating in high-temperature alloys.

Surface reactions on catalyst particles are of such importance that detailed experimental techniques have been developed to investigate their mechanisms. In a joint session with Symposium $\mathrm{R}$, J. Frenken (Leiden Univ.) described state-of-the-art high-pressure STM studies of the catalytic behavior of metal surfaces. Particularly interesting was the insight these studies provided into the mechanism by which Pd and Pt surfaces catalyze the oxidation of $\mathrm{CO}$ : When exposed to a $\mathrm{CO} / \mathrm{O}_{2}$ mixture, a very thin metal oxide layer is continuously formed and consumed, releasing $\mathrm{CO}_{2}$ as a reaction product. In contrast, reaction rates when oxygen is not being supplied are much slower. J. Janek and B. Luerssen (Justus Liebig Univ., Giessen) described photoelectron emission microscopy and scanning photoelectron microscopy studies that have elucidated the mechanisms by which oxygen is provided to electrode surfaces under solid-state electrochemical conditions, demonstrating, for example, that oxygen can be pulled from a yttriastabilized zirconia electrolyte at holes in a fully dense Pt electrode and showing subsequent surface diffusion of oxygen atoms over the Pt surface.

It is clear from these and other studies presented in the symposium that nanoscale understanding of the dynamics of surface reactions is providing critical information needed to address a variety of materials problems, including corrosion, environmental stability, energy storage, catalysis, and epitaxial film formation. The exciting new developments described in this symposium suggest that this area will continue to grow with the use of more sophisticated in situ instrumentation and techniques to model "real" environments.

Symposium Support: IBM T.J. Watson Research Ctr.

Through such events, a few strong supporters emerged on her behalf, Meyers said. She sent a resounding thanks to those along the way who looked beyond her gender and race, and allowed and encouraged her to excel. Finding the right mentors, Meyers said, also requires looking past the differences of others, as opportunity can come in many forms. Meyers said to guard and nurture the curiosity of young people, as that will carry them through.

"We can't afford to make mistakes," she said, "or we risk breaking someone's dreams."

\section{Surfaces and Interfaces Learn New Tricks}

A goal of Symposium Q was to assemble scientists who use a variety of approaches to control and tune the properties of sur- 
faces and interfaces. A unique aspect of this symposium was the cross-disciplinary nature of the talks that spanned materials involving block copolymers, polymer brushes, single-stranded DNA, thin-film silicon, colloidal particles, self-assembled monolayers, and small molecules. Applications including lithography, catalysis, microelectronics, wettability, and nanoscale chemistry, to name a few, were described.

S. Craig (Duke) provided a historical introduction to the field of polymer science, starting with Staudinger's discovery that polymers are long chain molecules. Craig created polymer brushes through molecular recognition of DNA strands. J. Youngblood (Purdue) showed that polyethylene glycol could be made hydrophobic by capping with fluorine groups. C. Nuckolls (Columbia) presented a potential alternative to thiol/Aubonding using carbene chemistry. DFT was used to support experimental evident of these new bridges. T. Russell (Univ. of Massachusetts, Amherst) showed that random copolymers grafted to surfaces can provide a neutral surface for controlling the orientation of block copolymer films so that cylinders are perpendicular to the substrate. This geometry is desirable for masking substrates for creating nanoscale features through optical lithography. By introducing a third monomer that is cross linkable, the random copolymer film $(5 \mathrm{~nm})$ was found to form a stable, neutral surface without the necessity of grafting to the substrate. There were also other highlights in the session on macromolecules, brushes, and soft matter. Synthetic, charged polymer brushes were controlled to extend and collapse by controlling solvent quality and introducing salt. An AFM tip could scan "through" a soft brush depending on the amplitude set-point. Another application of DNA as a smart material was demonstrated by creating a block copolymer of DNA and a hydrophobic block. These surfactant-like molecules absorbed at the air-water and oil-water interfaces, and maintained their recognition capabilities. Deposition on solid substrates was also demonstrated. DNA was also attached to polystyrene latex microspheres. Microspheres with complementary strands of DNA could be assembled either directly or by a third strand, resulting in selective and reversible aggregate formation. DFT was used to investigate the interface between metal surfaces and conjugated molecules. The total work function was extracted from its individual contributions. Phospholipid membranes were shown to recognize hydrophilic and hydrophobic regions of a photochemically patterned silane mono- layer surface. It was also revealed that dendrimers adsorbed to substrates produce smooth and hard metal films. The mechanism of this adhesion was unknown.

In sessions that focused on inorganic surfaces and the organic-inorganic interface, R. Nuzzo (UIUC), the keynote speaker, described the present state of soft lithography, nanotransfer printing, and decal transfer lithography. In a presentation involving live cells, it was shown that electric and magnetic files were used to organize yeast cells and particles into potential tissue scaffolds. Cell deposition by way of convection and sedimentation near a moving/evaporating contact line was demonstrated.

In a session on tunable and bio-active surfaces and their applications, switchable interfaces for biosystem control were demonstrated. In particular, SAMs were used to control microfluidic channels. Activation was turned on and off by the use of light, swelling of brush molecules, and heat. Switching of hydrophilic brush molecules as a function of temperature was used to control or prevent protein adsorption. In another presentation, it was shown that silicon wafers with pillars of controlled pitch can be described by simple wetting models and that they behave as superphobic surfaces. Electrowetting was used to move droplets, although pinning was a problem. Reversibility was achieved by rapidly heating the substrate. Applications to nanobatteries were described. The concept of electrowetting was utilized in digital microfluidics, as described in another talk. On-chip processing was demonstrated for moving blood and proteins, and for nanoscale mixing. Using silicone oil to "protect" the aqueous drop was noted as a key to rapid movement of the drop and limited contamination.

\section{Nanoporous Materials Designed for Catalysis, Sensing, and Gas Separation (See MRS Proceedings Volume 876E)}

In Symposium R, there was a cross section of the state of the art in the field of nanoporous and nanostructured materials for catalysis, sensor, and gas-separation applications, covering the range from basic to applied materials research and focusing on synthesis, functionalization, processing, characterization, computer modeling, and industrial development for applications.

In the last decade, intensive scientific research efforts have been made in the area of nanoporous materials. These materials can be prepared by a rich variety of techniques, either as granular solids formed by the aggregation of nanoparticles or in the form of monolithic nanoporous bodies (e.g., silica aerogels), formed by the growth of a porous oxide during anodic oxidation (e.g., porous $\mathrm{Al}_{2} \mathrm{O}_{3}$ from $\mathrm{Al}$ ), by the anisotropic etching of single-component single crystals (e.g., $\mathrm{Si}$ ), or by selectively removing one component from a solid solution (e.g., porous $\mathrm{Au}$ from $\mathrm{AgAu}$ ) or from a compound (e.g., porous $\mathrm{C}$ from $\mathrm{SiC}$ ). The properties of nanoporous materials are dominated by their extremely large specific surface areas, providing for properties that may significantly differ from those of bulk materials, and that may even acquire a characteristic size-dependence at the nanometer scale. Nanoporous materials are ideal candidates for surface-environment interactions, such as in gas sensing and heterogeneous catalysis. They are also under discussion as novel functional materials, where changes of the surface properties are induced by surface charging, either reversibly in situ or by ex situ charge injection. Although some nanoporous materials are already commercialized, the understanding of their formation processes is still in its infancy. An exciting new field is concerned with surface modification or functionalization, such as by atomic layer deposition.

Symposium Support: American Chemical Society-Petroleum Research Fund and PPG Industries.

\section{Synthesis, Characterization, and Applications of Magnetic Nanoparticles and Nanowires Presented}

(See MRS Proceedings Volume 877E)

Presentations in Symposium $S$ covered current multidisciplinary research on the synthesis, surface functionalization, characterization, and modeling of magnetic nanoparticles and nanowires. Highlights included the synthesis of self-assembled ordered nanostructures using chemical (C. Murray, IBM) and physical (D. Kumar, North Carolina A\&T State Univ.) methods, atomic-scale imaging of nanophase materials using a high-resolution electron microscope (S.J. Pennycook, ORNL), and targeted drug delivery by nanomagnetic particles (Q. Pankhurst, London Center for Nanotechnology; M. Abe, Tokyo Inst. of Technology).

Symposium Support: ARO and North Carolina AET State Univ.

\section{Nanostructured Diamond and Diamond-Like Materials Used for Micro- and Nano-Devices}

Symposium $\mathrm{T}$ focused on the science and technology of micro-, nano- , and ultrananocrystalline diamond and diamondlike thin films and their integration with dissimilar materials for application to a 

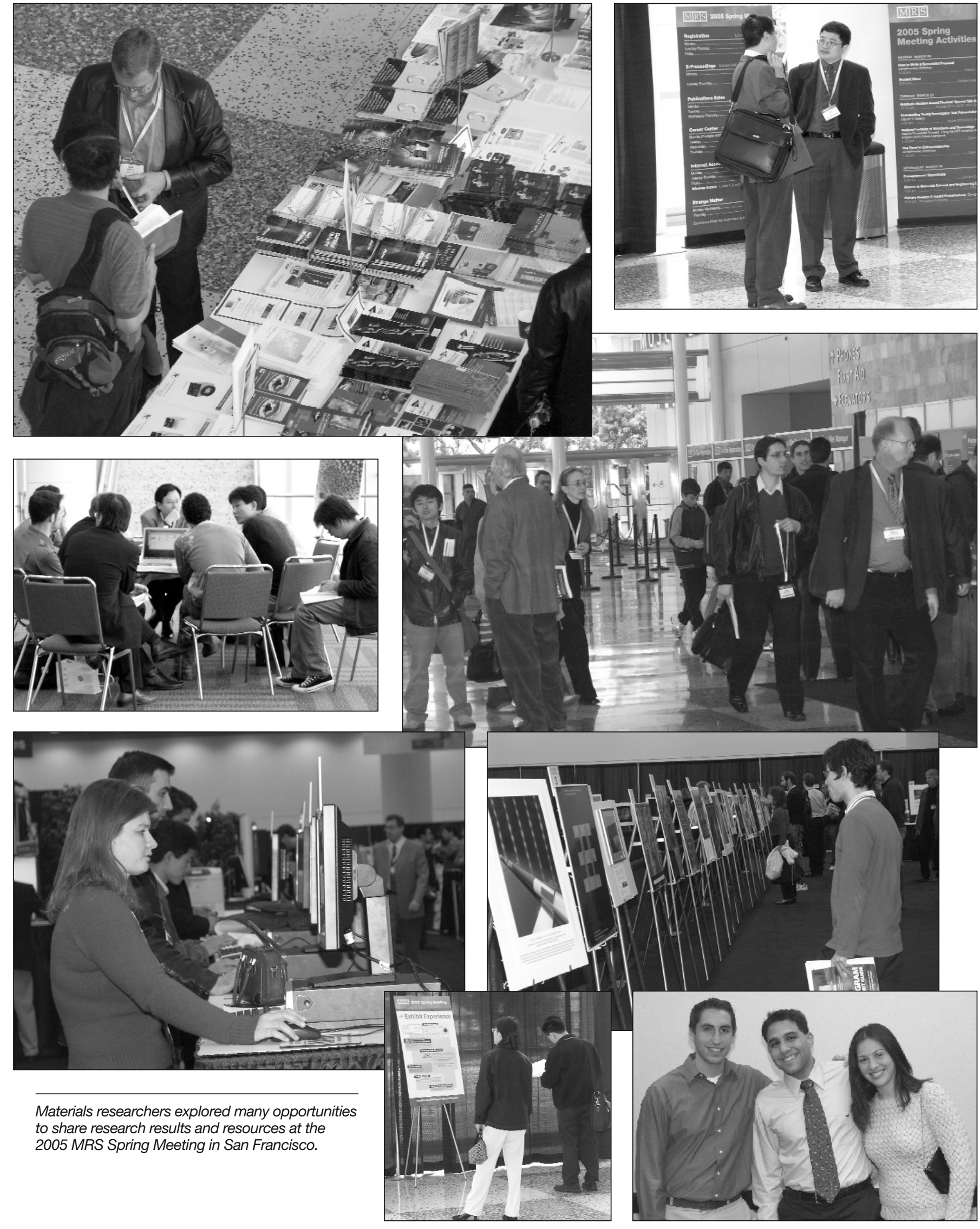
new generation of novel micro- to nanomultifunctional devices. Several presenters addressed issues related to the synthesis and characterization of different forms of diamond thin films and their properties and application to multifunctional microand nanodevices.

Highlights of invited talks included discussions of tetrahedral amorphous carbon films for MEMS; tribological studies of diamond surfaces at the nanoscale; a critical comparison of the mechanical properties of nanocrystalline diamond and tetrahedral amorphous carbon films with a view to applications to MEMS and NEMS; the potential use of carbon-based materials in vacuum thermionic energy conversion; the properties of new ultrananocrystalline diamond thin films that exhibit a unique combination of mechanical, tribological, electronic transport, and biocompatible characteristics; in situ TEM studies of mechanical properties of different forms of diamond films and comparison with the performance of Si as a MEMS material; studies of immobilization of redox enzymes on functionalized ultrananocrystalline diamond surfaces; interfacing microelectronics to biological systems using diamond films; functionalization of diamond surfaces and applications to biosensors; sulfur doping of micro- and nanocrystalline diamond films; and plasma systems for deposition of large-area diamond films critical to fabrication of MEMS and NEMS devices in an industrial environment.

Highlights of contributed talks included a discussion of the science and technology of ultrananocrystalline diamond as a hermetic/bioestable encapsulation coating. Such a coating is being considered for use with a Si microchip implanted in the eye as a critical component of an artificial retina to restore sight to people blinded by retina degeneration. Other highlights included the electronic structure of diamondoids using synchrotron radiation; bonding and structure of hydrogenated amorphous carbon by resonant Raman spectroscopy; diamond MISFETs fabricated from homoepitaxial B-doped diamond on (100)- and (111)-oriented substrates; and nanocrystalline diamond-based nanomechanical resonators, including unique integration of oxide piezoelectric thin films with nanocrystalline diamond layers.

Symposium Support: ARO.

\section{Control of Carbon Nanotubes Enhanced}

Symposium U, Science and Applications of Carbon Nanotubes, was a multidisciplinary gathering that covered a wide spectrum of topics ranging from materials synthesis, processing, and char- acterization to devices and applications. Use of alcohols as carbon feedstock for dense arrays of SWNTs was discussed by S. Maruyama (Univ. of Tokyo). Efforts in substitutional doping by boron and interstitial doping by alkali metals as well as characterization of such materials by EELS were presented by T. Pichler (IFW, Dresden). DNA has been used to both position nanotubes in precise locations for devices (R. Naaman, Weizmann Inst.) and as means for sorting them by diameter (M. Zheng, DuPont). Macroscale materials based on nanotubes can be obtained by creating composites with polymers (J. Coleman, Trinity College Dublin) and spinning of nanotube fibers (I. Kinloch, Cambridge).

In addition to Raman-based characterization, light absorption and luminescence can identify individual nanotube species. Particularly interesting were reports of these measurements in solid-state devices (J. Lefebvre, NRC, Canada) and in solution at high magnetic fields (S. Zaric, Rice). S. Kalinin (ORNL) described highfrequency scanning probe techniques that look at nanotubes in devices and polymer composites at the megahertz time scale.

Applicability of carbon nanotubes was discussed by several speakers. G. Duesberg (Infineon) described advantages that nanotubes may hold in the semiconductor industry, including in both transistor and interconnect layers. Advances in local chemical doping of nanotube transistors and movable infrared light sources based on nanotubes were presented (J. Chen, IBM). V. Bouchiat (CNRS, Grenoble) incorporated nanoparticles in the gate stack of nanotube transistors to demonstrate single-electron memory. The progress toward field-emitters based on carbon nanotubes was reported by M. Doytcheva (Philips).

Symposium Support: FEI Company Latin America, JEOL USA, Philip Morris USA, Raith USA, and Sociedad Mexicana de Nanociencias y Nanotecnologia.

\section{Rare-Earth-Doped Semiconductors Cultivated for Optoelectronics} (See MRS Proceedings Volume 866)

Symposium V provided a forum to highlight recent advances in the field of optoelectronic applications of rare-earthdoped semiconductors. Traditionally, the motivation for research on these materials has been the development of electrically driven optical amplifiers and sources at telecommunications wavelengths in the infrared range. Important advances were reported in erbium-doped nanostructured $\mathrm{Si}$ and $\mathrm{SiO}_{2}$ materials. The use of Si nanocrystals has led to highly reduced thermal quenching of infrared luminescence. Z.F. Krasilnik (Russian Academy of Sciences) reported on erbium-doped silicon multilayer structures based on MBE. This approach permitted optimization of the excitation of erbium ions for LEDs. Data from the LEDs support the feasibility of achieving an erbium-doped silicon laser in the future. A. Polman (AMOLF) gave an overview of microstructured $\mathrm{Si}$ and $\mathrm{SiO}_{2}$ materials. He reported on stimulated infrared emission in erbium-doped microcavity structures that were produced through the use of lithography and etching. A number of other talks focused on optical gain in erbium-doped silicon-rich oxide materials (M. Forcales, ANU; L. DalNegro, MIT). While this issue is critical for laser development, results indicate that further study is required.

In recent years, there has been considerable activity in rare-earth doping of wide-bandgap semiconductors such as $\mathrm{GaN}$ and SiC. Several talks described photoluminescence and electroluminescence at visible wavelengths (V. Dierolf, Lehigh; E.E. Nyein, Hampton; S. Gallis, SUNY-Albany). C. Munasinghe (Univ. of Cincinnati) reported on an interrupted MBE growth technique for optimizing the visible luminescence from europiumdoped $\mathrm{GaN}$ thin films. This technique may have important implications for preparing better phosphors used in visible displays. Two talks described methods for producing rare-earth-doped GaN powders. Preliminary data indicate high potential for novel photonic applications.

Symposium Support: ARO.

\section{Still Much to Learn about CMP Despite Widespread Use}

(See MRS Proceedings Volume 867)

While now in widespread use in IC fabrication, CMP still receives a lot of attention from fundamental researchers as well as integration engineers. CMP continues to pose substantial challenges, including its use for virgin silicon preparation, shallow trench isolation, and dielectric polishing as well as for copper and low-dielectric-constant (low- $\kappa)$ metal interconnects. The large number of variables to be considered was reflected in Symposium W on CMP: Integration, Technology, and Reliability.

Topics ranged from studies of consumables such as pads and slurries and their mutual interactions to novel polishing methods such as electrochemical-mechanical planarization. The role of the different slurry constituents such as abrasives, including silicon, aluminum, cerium, and zirconium oxides, and additives such as 
oxidizers, corrosion inhibitors, and surfactants was extensively discussed.

Insight into the interaction between pad and substrate was presented; by using dual-emission laser fluorescence, the extension of the slurry film can be photographed in situ on a microscopic scale. M. Moinpour (Intel) discussed the use of AFM to study the adhesion between slurry particles and various substrates. Several contributions were dedicated to modeling CMP.

N. Gitis of the Center for Tribology presented a standard procedure for qualifying conditioners, pads, and slurries using the frictional and acoustic-emission characteristics of these consumables. The research group of A. Philipossian (Univ. of Arizona) also utilized the coefficient of friction as a parameter to investigate pad lifetime, slurry type, conditioning method, and delamination. Delamination is especially important for the integration of fragile low- $\kappa$ materials. In another presentation, an overview was given of different failure mechanisms in $\mathrm{Cu} /$ low- $\kappa$ stacks during CMP. The research group of R. Dauskardt (Stanford) investigated the influence of the slurry characteristics (e.g., $\mathrm{pH}$, oxidizer) on the crack growth in low- $\kappa$ films.

Symposium Support: Seagate Technology.

\section{Versatility of Solvothermal Synthesis Shown}

(See MRS Proceedings Volume 878E)

Presentations in Symposium Y covered solvothermal synthesis, characterization, and property evaluation and applications of powders and single-crystal materials. In solvothermal processing, solvents such as water or organic alternatives such as methanol, ethanol, or polyol are used in the formation of materials shaped as powders, for example, typically conducted at or below the supercritical temperature of the solvent. T. Adschiri (Tohoku Univ.) presented an invited talk on supercritical hydrothermal synthesis of organic-inorganic hybrid nanoparticles. By mixing aqueous salt solutions and organics with high-temperature water, he was able to rapidly heat the solutions to form nanoparticles modified with organic materials. In another invited presentation, G. Patzke (ETH, Zurich) described solvothermal pathways for the synthesis and morphological control of transitional metal oxides for future nanotechnology applications. She described a unique solvothermal method with an in situ x-ray diffraction capability to characterize the materials as they are formed.

There were several presentations on
$\mathrm{ZnO}$, which is an important material with interesting optical properties (such as UV lasing behavior) and an important candidate for gas sensors. For example, D. Lundeen and F.F. Lange of UCSB described the lateral epitaxial growth of $\mathrm{ZnO}$ with citrate ions to reduce defects in $\mathrm{ZnO}$ films. J. Choy (Ewha Womans Univ., Seoul) described precise control of $\mathrm{ZnO}$ morphology by solvothermal synthesis. G. Demazeau (Univ. of Bordeaux) described a solvothermal process for cubic boron nitride and new phyllosiloxide (i.e., layered oxides) materials synthesis. S. Mathur (Leibniz Inst. of New Materials) described the solvothermal synthesis of oxide materials with precise composition and morphology by utilizing molecular compounds. M. Yoshimura (Tokyo Inst. of Technology) showed that electrosynthesis is useful to produce carbon nanoshells, which can be used to encapsulate metal nanoparticles for possible biological applications. R. Riman (Rutgers) demonstrated the versatility of the solvothermal method in the growth of various ceramic coatings. S. Komarneni (PSU) showed the novelty of the use of microwave and/or a biomolecule-assisted solvothermal process for the controlled and rapid synthesis of many types of metal and semicon-

\section{Hwang Describes Atomic Control of Electronic Structure at Complex Oxide Heterointerfaces}

Harold Y. Hwang (University of Tokyo), the 2005 MRS Outstanding Young Investigator, presented his award talk in a special session at the MRS Spring Meeting. He said that perovskite oxides exhibit a wide range of physical

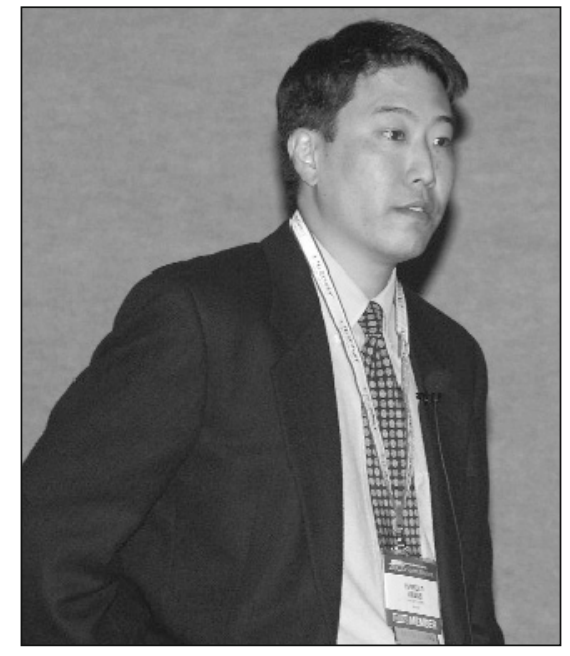

Harold Y. Hwang of the University of Tokyo is the 2005 MRS Outstanding Young Investigator. properties, from insulators to superconductors, incorporating magnetism and coupling to phonon instabilities. The close lattice match between many perovskites raises the possibility of growing epitaxial thin-film heterostructures with different ground states that may compete or interact. Among the issues that arise, many are generic to all heterointerfaces, such as the stability of dopant profiles and diffusion, interface states and depletion, and metastable growth and extended defects. The increased structural complexity over conventional semiconductors leads to a wider variety of experimentally realized interface structures, which are a strong function of the growth kinetics and defect chemistry, Hwang said.

For highly kinetic growth, atomically abrupt perovskite interfaces and superlattices can be produced allowing direct control of the termination layer at the interface. The composition, valence, and excited states can be measured on the atomic scale using spatially resolved electron energy-loss spectroscopy, revealing natural charge distribution lengths and non-bulk-like electronic structure. One important manifestation of atomically abrupt interfaces is the interface charge arising from polarity discontinuities analogous to that in $\mathrm{GaAs} / \mathrm{Ge}$ interfaces for various crystalline orientations. This manifestation enables a novel approach to creating new two-dimensional charge states; it also represents an important issue that must be controlled for device applications.

Another intriguing issue Hwang discussed is the charge structure associated with Mott insulator/band insulator interfaces, which brings up the question of how one should consider the correlated equivalent of band bending. This semiconductor concept is based on the validity of rigid single-particle band diagrams, which are known to be an inadequate description for strongly correlated electrons. In addition to presenting an interesting scientific challenge, this underlies attempts to develop new applications for doped Mott insulators in device geometries. One unique manifestation of this issue is in rectifying manganite-titanate heterojunctions, for which the charge distribution can be modified by a magnetic field, reflecting the strong charge-spin coupling arising from the double-exchange mechanism. 
ductor nanowires and nanorods. The many exciting results presented in this symposium clearly demonstrate that the "solvothermal" process is a versatile lowtemperature synthesis and processing technique.

Symposium Support: PSU-Materials Research Institute.

\section{Chemical Methods Feed Advancement of Nanomaterials}

(See MRS Proceedings Volume 879E)

The objective of Symposium Z, Chemistry of Nanomaterial Synthesis and Processing, was to bring together scientists and engineers with diverse backgrounds to discuss the most recent advances in the new chemical methodologies being developed for nanomaterial synthesis and processing. The symposium included sessions on 1D nanostructures that focused on nanotube and nanowire synthesis, assembly, and applications. H. Dai (Stanford) described recent work in measuring ballistic electron transport in metallic and semiconducting nanotubes and the implications of transport in pushing the performance limit of nanotube transistors. As characterization is becoming more and more important in understanding and achieving structural control of nanomaterials, Z.L. Wang (Georgia Tech) described the subtleties of the structural analysis of nanobelts by TEM and its broad application in determining materials attributes such as planar defects and twin/bicrystal structures. P. Yang (UC-Berkeley), in his presentation, inspired substantial interest on the synthesis and optical properties of nanowires.

The largest number of presentations dealt with the synthesis, assembly, and applications of nanocrystals and related surface and interfacial chemistry. New results in the area of the growth of highly branched nanostructures were given in separate talks by P. Alivisatos (UCBerkeley) and J. Liu (SNL). Alivisatos described how to control the growth and branching of CdSe/CdTe nanoparticles in their quantum-confinement size regime, while Liu described how growth modifiers can be used to manipulate and control the formation of hierarchical nanostructures of $\mathrm{ZnO}$. Such complex structures will likely become hot topics in the field of colloidal nanostructures, and they will receive a considerable amount of attention in gas-phase nanostructures as well. Complex nanostructures were also explored through other means in the field of colloidal nanocrystals. Y. Xia (Univ. of Washington) described how complex nanostructures could be made through solid-state chem- ical reactions. Similar approaches were discussed by Alivisatos.

Applications of nanomaterials are undoubtedly of interest for the community. J. Rochenberge (Kovio, California) described how colloidal nanocrystals can be used as "inks" for the fabrication of ICs. D. Awschalom (UCSB), P. Avouris (IBM), M. Bawendi (MIT), D. Crooks (Texas A\&M), H. Park (Harvard), D. Zhao (Fudan Univ.), and many others presented their recent results on exploring nanostructures for a variety of applications.

The environmental implications of nanostructures are becoming more and more of a concern, especially in the general public. V. Colvin (Rice) reported that proper surface modification of nanostructures will likely become the key to addressing some of these issues.

Symposium Support: NanoLab, Nanomaterials $\mathcal{E}$ Nanofabrications Laboratories, and SNL.

\section{Dynamic Reorganization Yields Novel}

\section{Responsive Properties of Nanomaterials}

Self-assembly has enabled a rich variety of nanoscale-structured materials with exquisite hierarchical features. Only recently has it become possible to program the components to dynamically reorganize into specific architectures, yielding novel responsive physical, mechanical, and chemical behavior in structured materials. Symposium AA highlighted some of the advances in this burgeoning area, covering the design and synthesis of components; the assembly process; and the resulting optical, magnetic, electrical, biocompatible, and structural properties. A tutorial on the subject was offered by Y. Xia (Univ. of Washington) and Z.L. Wang (Georgia Tech). The symposium presentations opened as a joint session with Symposium Q, covering topics on the self-assembly of magnetic nanoparticles and nanostructures. C. Murray began the session with a presentation outlining the nanoparticle superlattice structures developed at IBM. T. Russell (Univ. of Massachusetts, Amherst) and Z. Wang (Georgia Tech) then discussed the fabrication of precisely defined nanowires and nanobelts. A variety of novel nanosystems were presented in the sessions on building blocks and new structures. The blocks being studied and assembled by different groups range from molecules to nanoparticles, microemulsions, and droplets.

The development of nanostructures interfacing biological components is a new and rapidly developing area. The presentations in this session included the use of live cells as directors for sol-gel coatings, the morphosynthesis of patterned crystals, the layer-by-layer assembly of biocompatible membranes and artificial retinas, and the assembly of multicomponent nanowires by biological interactions.

Another area outlined in the symposium was the formation of hybrid and dissipative structures. A session on this topic was opened by T. Kato (Univ. of Tokyo), who demonstrated how anisotropic ionconductive materials can be fabricated by molecular self-assembly. A variety of periodic and functional structures assembled or manipulated by electric fields or temperature gradients were reported.

Functional nanostructure-based devices were presented in a session on responsive materials and nanoarrays and tunable optics. The structures presented included responsive liquid crystals, novel "colloidosome" capsules, responsive patterned phospholipid bilayers, photonic and antireflective coatings, and photonic switches based on periodic arrays of liquid-crystal droplets. The session on electronic and magnetic behavior addressed the issue of functional building blocks. N. Halas (Rice) reported the development and application of arrays of core-shell particles with tunable plasmonic properties. Other structures of interest included conductive polymers and piezoelectric, magnetic, and conductive assemblies from nanowires.

Symposium Support: AFRL, ARO, and SNL.

\section{Experiment and Modeling Combined in Study of Nanostructure Effects on Mechanical Properties}

(See MRS Proceedings Volume 880E)

The aim of Symposium BB, Mechanical Properties of Nanostructured Materials: Experiments and Modeling, was to bring together experimentalists and theorists to discuss nanoscale deformation mechanisms that lead to significantly enhanced mechanical properties. Various novel means of characterizing deformation using x-ray diffraction or electron backscatter diffraction were presented. There were also several talks on deformation mechanisms within grains and the cooperative deformation of grains.

Structure-property relationships in biomaterials were another focus area. E. van der Giessen (Univ. of Groningen) discussed a new model for non-affine stiffening in actin networks, D. Raabe (MPI, Dusseldorf) showed how the texture of biological materials contributed to their mechanical function, and H. Gao (MPI, Stuttgart) presented a model of flaw tolerance achieved through hierarchical structure. Structure was also shown to be important in the controlled synthesis of $\mathrm{ZnO}$ nanorings and nanobows (W. Hughes, Georgia Tech). 
Modeling of nanoscale deformation was a highlight of the Symposium. D. Wolf (ANL) showed how atomistic methods could be used to develop a mesoscale model of creep and grain growth at long time scales. H. Van Swygenhoven (Paul Scherrer Inst.) detailed dislocation emission from grain boundaries and twin boundaries, and P. Gumbsch (Univ. of Karlsruhe and Fraunhofer-Institut für Werkstoffmechanik) demonstrated how glissile dislocation junctions can lead to the creation of new Frank-Read sources. Synthesis and novel experimental methods were also major areas of interest. The role mechanics plays in nanoimprinting was described (J. Pethica, Trinity College Dublin), and a method to test thin films in both tension and compression produced surprisingly different Bauschinger effects in different materials (J. Vlassak, Harvard). Fatigue results for nanostructured materials were also described by several presenters.

Symposium Support: Hysitron.

\section{Modeling Nonlinear Behavior Important} for Coupling Functional Properties (See MRS Proceedings Volume 881E)

Modern sensor and actuator applications are based on coupling phenomena such as thermoelasticity, piezoelectricity, pyroelectricity, electrostriction, magnetostriction, shape-memory effects, and magnetoelectricity. Occasionally idealized as linear, the true nature of these phenomena is nonlinear. The intent of Symposium CC was to offer a platform for the presentation and discussion of advanced modeling and simulation methods for coupling phenomena. To help put the models and simulations in context, presentations on experimental research were given as well.

The type of materials addressed ranged from magnetic shape-memory alloys to ferroelectric ceramics, both in bulk and thin-film configurations. Newly designed materials were presented, including shapememory honeycombs and multiferroic magnetoelectric composites. Experimental work employing Lorentz microscopy, SPM, nanoindentation, and advanced diffraction methods as well as sophisticated macroscopic characterization techniques was featured. Presentations on modeling covered the entire spectrum of length scales, from first-principles atomistic calculations and phase-field simulations through advanced micromechanics to nonlinear continuum methods.

A major conclusion inferred from this symposium is that finite-element-based methods for the nonlinear behavior of smart materials and devices have become widely available, whereas a few years ago, no such tools were at hand for scientists and engineers.

\section{Heat and Mass Follow Interesting Paths on the Nanoscale}

Symposium DD, Heat and Mass Transport at Nanoscale-From Fundamentals to Devices, focused on the issue of tailoring transport by nanostructured systems and structures. For such systems, the classical picture of a diffusive heat-flow mechanism is often not applicable because the phonons or electrons that carry heat have mean free paths comparable to, or smaller than, the nanoscale feature size. In the case of the mass-flow problem, the NavierStokes equation with no-slip boundary conditions is often inadequate. J.J. de Pablo (Univ. of Wisconsin) and A. Majumdar (UC-Berkeley) analyzed the flow of biomolecules by nanochannels and demonstrated potential for nanofluidic logic devices. S. Choi (ANL) opened discussion on the most hotly debated topic, the unusually large thermal-transport enhancement observed in nanofluids, that is, colloid suspensions of solid nanoparticles. In this context, S. Volz (CNRS, France) proposed a "giant thermal conductance effect" between polar nanoparticles. Discussion of carbon nanotubes focused on their behavior in composites and suspensions and the role of the thermal boundary resistance on the effective composite thermal conductivity. D. Cahill (UIUC) described experimental studies on the thermal resistance of individual interfaces between soft and hard solids and demonstrated the importance of nonharmonic effects. In the application area, N. Halas (Rice) reported the successful treatment of cancer in mice by hyperthermia induced by local tissue heating due to laser-excited silica-gold nanoshells.

\section{From Electrons to Steel Forming- All Scales Necessary to Model Mechanical Behavior of Materials (See MRS Proceedings Volume 882E)}

Symposium EE, Linking Length Scales in the Mechanical Behavior of Materials, showed that capturing the complexity of the multiscale origins of mechanical properties in modeling more and more spans all length and time scales, from the electronic origins of mechanical behavior to industrial forming processes of materials. D. Sen (OSU) demonstrated, at the smallest length scale, the link between electronic structure and grain-boundary brittleness in $\mathrm{Ni}_{3} \mathrm{Al}$ and thus the ultimate foundation of mechanical properties in the electronic structure of materials. B.P. Uberuaga (LANL) discussed the increasingly popular accelerated molecular dynamics meth- ods, which can extend the simulation times accessible to molecular dynamics from nanoseconds into the realm of seconds. By coupling these methods with kinetic rate theory, the wide variations in time and length scales involved in microstructural changes due to radiation damage in ceramics can be modeled. M.O. Robbins (JHU) presented a hybrid atomistic/continuum method, where areas of special interest such as interfaces and high-stress regions are described by molecular dynamics in an otherwise continuum model. Such models are especially useful for systems with potentially singular finite element stresses. Discussed examples ranged from fluid flow to solid contacts. D. Raabe (MPI, Düsseldorf) demonstrated, on a larger length scale, coupling of the microstructure to the macroscopic level by including a detailed dislocation-density model and grain-boundary mechanics in the constitutive equations of a finite element crystal plasticity model, resulting in a strongly improved description of plasticity during large-strain metal deformation.

Symposium Support: LLNL.

\section{Advanced Materials and Optoelectronic Technologies Enable Compact Development and Widespread Utilization of Lidar Instruments (See MRS Proceedings Volume 883)}

Laser remote sensing is a powerful technique with a wide range of applications in fields as diverse as atmospheric studies and climate research, marine ecology, weather forecasting, space exploration, air travel safety and efficiency, military target identification, and countermeasures, thus addressing significant societal needs. Over the past three decades, many lidar (laser radar) instruments have been developed and their capabilities demonstrated from ground-based, airborne, and space-based platforms. The main goal of Symposium FF was to gather scientists and technologists from different disciplines to create new avenues for advancing the practice and progress of laser remote sensing and to enable new sensing paradigms by applying advanced materials, semiconductor devices, and modern optoelectronic technologies. For example, advanced devices developed by the telecommunications industry can play an important role in addressing the increasing demand for more efficient and compact lidar systems. Fiber-optics lasers described by N. Peyghambarian (Univ. of Arizona) and MEMS-based semiconductor lasers reported by O. Solgaard (Stanford) are examples of such devices. The potential of fiber optics and photonic devices in the advancement of laser remote sensing was 
further elaborated in a panel discussion led by P. Moulton (Q-Peak Inc.) and N. Barnes (NASA Langley Research Center). They noted the use of compact, efficient, and inexpensive optical components for telecommunications and the effect they are having on the growing utilization of laser remote sensing techniques.

The reliability of lidar instruments is dependent on the high-power laser diodes used for pumping their transmitter lasers. For lidar instruments operating in space, the reliability and lifetime of laser diodes are particularly critical because of the high development and launch costs of instruments and their inaccessibility once in space. In fact, NASA has identified laser diodes as one of the major risk areas in the deployment of laser systems in space. H. Leidecker (NASA Goddard Space Flight Center) presented the results of an investigation on the recent laser failure onboard an orbiting lidar satellite (IceSat). E. Stephens (Northrop Grumman) and B. Meadows (NASA Langley Research Center) explained the failure mechanisms of laser diodes and offered solutions for improving their lifetime and reliability. A special topics presentation by Leidecker provided a more in-depth understanding of laser diode reliability issues. As a counterpart of NASA, the European Space Agency was represented by D. Morançais, from EADS Astrium, who described the latest advances of ALADIN (the atmospheric laser Doppler instrument), the first European lidar in space.

Any improvement in efficiency of detection devices will directly translate to a lower required transmitter laser power, resulting in more compact and efficient lidar devices. Novel Geiger-mode avalanche photodiode arrays developed at MIT's Lincoln Laboratory (presented by R. Heinrichs) can drastically affect the imaging lidar applications. A combination of the high speed and high efficiency of these devices will enable several military and space applications that require high spatial resolution and a short frame-refresh time.

A session on the future of laser remote sensing and applications concluded this symposium. This session also included presentations addressing NASA's lidar needs in meeting the objectives of the U.S. space program focusing on staffed missions to the moon and Mars.

Symposium Support: NASA Langley Research Ctr. and SINTEF Norway.

\section{Hydrogen Storage Alternatives Compared \\ (See MRS Proceedings Volume 884E)}

A hydrogen storage system is considered the most crucial component of the "hydrogen economy." The current hydrogen storage technology based on pressurized gas tanks does not meet the requirement of a 300 mile driving range for transportation applications. Therefore, significant global efforts have been devoted to developing a practical solidstate hydrogen storage material. The aim of Symposium GG on this topic was to serve as a forum for discussions on fundamentals and applied aspects of hydrogen storage materials, providing an understanding of the challenges and opportunities for the materials research community. The technical sessions of the symposium focused on the hydrogen storage characteristics of metalorganic frameworks (MOFs), ammonia borane complexes, complex chemical hydrides, metal and alloy hydrides, and carbonaceous materials, as well as the production of hydrogen by the conversion of organics and by the splitting of water using solar cells. Several diagnostic tools for evaluation of hydrogen storage materials were also presented. DOE, which reported a major focus on the development of solidstate hydrogen storage materials, provides guidance and basic requirements for the new storage materials. One of the major components of the program is to develop breakthrough materials to meet the challenging requirements of hydrogen storage for transportation applications.

Results on hydrogen storage characteristics of light metal nitrides were reported. Modifying $\mathrm{Mg}\left(\mathrm{NH}_{2}\right)_{2}$ by the addition of light metal hydrides $(\mathrm{LiH}, \mathrm{NaH}$, and $\mathrm{MgH}_{2}$ ) provides over $6 \mathrm{wt} \%$ hydrogen sorption below $150^{\circ} \mathrm{C}$. The hydrogen sorption characteristics of most available nitrides, amides, and imides of light metals, including binary and ternary nitrides, were presented. The work has shown the promising potential of light metal nitrides for hydrogen storage. Several experimental and detailed theoretical works on catalyzed sodium alanate were presented. Despite extensive work in this area, no clear understanding of the mechanism of the catalysis is available. Most reports show the existence of catalysts such as $\mathrm{Ti}$ metal on the surface of alanate particles with no substitution in the lattice sites. Recent work on reversible alkali borohydrides have shown close to $6 \mathrm{wt} \% \mathrm{H}$ sorption. The kinetics of the process is still very slow, and the screening of catalysts to improve the hydriding-dehydriding rate is under intense investigation. Partial reversibility of ammonia borane complexes was reported. Although this is a significant achievement, the reversible capacity is still less than $2 \mathrm{wt} \%$, and the detection of ammonia during heat treatment above $150^{\circ} \mathrm{C}$ was mentioned. The chemistry of borazine, cycloborazane, and polyborazylene was also presented. Hydrogen storage in clathrates with a sorption capacity of up to $3.77 \mathrm{wt} \%$ at ambient pressure was reported with a fast preparation of clathrates under high hydrogen pressure and low temperature. A new MOF based on a copper organometallic compound (MOFs 504) with a mesoporous structure and a hydrogen sorption capacity of $2.5 \mathrm{wt} \%$ at $77 \mathrm{~K}$ and 1 bar was reported.

A comprehensive analysis of a highpressure alloy tank (i.e., a pressurized tank that contains hydrogen storage alloys) based on an optimized Ti-Cr-Mn alloy $\left(\mathrm{AB}_{2}\right.$ Laves phase) was presented. The system has fast hydrogen delivery and good performance in a wide temperature range (from $-40^{\circ} \mathrm{C}$ to $70^{\circ} \mathrm{C}$ ). The current technology provides only $1.9 \mathrm{wt} \%$ hydrogen storage capacity. Despite the low gravimetric hydrogen storage capacity of the alloy, the tank is still significantly better than a conventional pressurized gas cylinder. The hydrogen absorption-desorption property of organic hydrides was also presented. The process has been optimized for a decalene system that provides close to $100 \%$ conversion efficiency below $200^{\circ} \mathrm{C}$.

Several diagnostic tools were discussed. Among them, low-energy ion-scattering direct recoil spectroscopy, vibrational spectroscopy, and neutron-diffraction techniques were considered useful for the evaluation of H-storage materials. Neutron diffraction of hydrogen-absorbing alloys provides useful parameters (e.g., $\mathrm{H}$ sites, anisotropic volume change during hydrogenation, phase changes, strain deformation, and particle size) that can be deduced from the neutron-diffraction data.

Several works on hydrogen production were presented. The conversion of organics and biomass to hydrogen by supercritical water was presented. The biomass conversion system is currently scaled up to $100 \mathrm{~kg}$ per hydrogen plant. Further optimization of this system for higher energy efficiency and applicability to different feedstock are in progress.

The conversion of glucose to hydrogen without the formation of tar and char with good efficiency was also reported. Hydrogen production from the hydrolysis of $\mathrm{NaSi}$ was also reported. The hydrolysis byproduct is nonreversible sodium silicate.

Symposium Support: Toyota Motor Sales, USA.

Nanotechnology Meets the Science and Technology of Integrated Nanosensors

Symposium $\mathrm{HH}$ served as a focal point for developers and advanced users of nanostructured sensors of different types. The integration, on a single platform, of 
sensors sensitive to magnetic, infrared, chemical, or biological stimuli was the main goal. The symposium provided an overview of recent advances and emerging new sensor technologies in the fields of science, engineering, and medicine.

The topic of integrated nanosensors was reviewed through the perspectives of, respectively, DoD, NSF, and DOE. According to H. Weinstock (AFOSR), who gave an overview of the various DoD sensor programs, the future activities must be concentrated on the development of "all-knowing" multiple sensing capabilities and their integration. The key to promoting innovative sensor research is collaboration among different science and technology disciplines. J.M. Hicks (NSF) stressed the importance of clear targets in the basic research projects of sensor research supported by the National Science Foundation, as well as the importance of feedback and adaptive sensing. T. Michalske (SNL) discussed the perspectives from DOE for integrated nanosensors in the fields of energy, security, and the environment.

Many presentations dealt with microcantilevers for chemical and biological sensing. M. Hegner (Basel Univ.) demonstrated that cantilever arrays offer a novel parallel label-free approach, with potential applications such as antibiotic testing, spore detection in food industry, and biodestruction of materials. A. Boisen (Denmark Univ.) reviewed the potential of cantilever-array sensing devices with integrated readout and electronics. The use of polymers makes the fabrication process simple, cheap, and flexible. Readout of cantilever arrays based on the optical-beam deflection method was discussed by J. Tamayo (CNM, Madrid).

Other areas of research receiving extensive coverage in the symposium were sensors based on nanoparticles and nanowires. The characterization of chemical microsensors for volatile organic compounds, based on titania deposited on microfabricated platforms, was discussed by P. Milani (Milano Univ.). Magnetic microparticles functionalized with antibodies can be used for ultrasensitive detection of proteins and nucleic acids, as was shown by D. Georganopoulou (Northwestern). Addressable nanowires using integrated nanosensor arrays and nanowire FETs were reported in a number of talks.

A smaller number of presentations focused on sensors based on Si photonic crystals, as described by M.J. Sailor (UC-San Diego); SWNT arrays, as demonstrated by A. Star (Nanomix,
California); and spintronic devices using the momentum transfer mechanism, as developed by J. Daughton (NVE Corp., Minnesota). A powerful approach to the detection of chemically diverse analytes is the colorimetric sensor array, as presented by K. Suslick (UIUC).

In the session on physical sensors, the fundamental issues of implementing infrared imaging with small-scale focalplane arrays were reviewed by G.J. Brown (AFRL), while in the session on chemical sensors, W.C. Trogler and A. Kummel (UCSD) discussed the possibilities offered by chemosensors fabricated from metallophthalocyanine nanofilms on interdigitated electrodes and FETs.

The last two sessions of the symposium stressed the importance of sensor integration and applications. A new technology platform of fluidic-photonic ICs was presented by Y.-H. Lo (UCSD). B. Kasemo (Chalmers Univ., Gothenburg) described cell-mimicking membranes, active nanoparticles for sensing, and microfabricated structures that mimic sharkskin surfaces. The interaction between electronics and biology-in particular, a neuroelectronic junction based on microelectronic Si chips-was discussed by S. Borghs (IMEC, Leuven).

The symposium showcased advances obtained recently through the use of nanostructured or nanocomposite sensors. The integration of multiple sensors on a single platform will be the major challenge for the future development of nanostructured sensor devices.

\title{
MRS Materials Research Society
}

\section{Upcoming MRS Meetings \& Workshops}

\author{
Organic Microelectronics Workshop \\ July 10-13, 2005 - Newport, Rhode Island \\ www.mrs.org/meetings/workshop
}

Three-Dimensional Multifunctional Ceramic Composites Workshop

October 3-5, 2005 • Urbana, IL

www.mrs.org/meetings/workshop

2005 MRS Fall Meeting

November 28-December 2, 2005 - Boston, MA

www.mrs.org/meetings/fall2005/

\section{Upcoming MRS-Endorsed Meetings}

\section{ICMAT 2005 and IUMRS-ICAM 2005}

July 3- 8, 2005 - Singapore

www.mrs.org.sg/meeeting_future.html

16th International Conference on Electronic Properties of Two-Dimensional Systems (EP2DS-16)

July 10-15, 2005 - Albuquerque, New Mexico

ep2ds-16.sandia.gov

Gordon Research Conf.: Combinatorial \& High Throughput

Materials Science III

August 14-19, 2005 • Oxford, UK.

www.grc.org/programs/2005/combhigh.htm

SMMIB: International Conference on Surface Modification of Materials by Ion Beams

September 4-9, 2005 - Kusadasi, Turkey

www.smmib05.net

Materials Research Society

506 Keystone Drive, Pittsburgh, PA 15086-7573 USA

Tel. 724-779-3003 • Fax 724-779-8313

E-mail: info@mrs.org • www.mrs.org 